\title{
A charge preserving scheme for the numerical resolution of the Vlasov-Ampère equations.
}

\author{
Nicolas Crouseilles* Thomas Respaud ${ }^{\dagger}$
}

January 11, 2011

\begin{abstract}
In this report, a charge preserving numerical resolution of the 1D Vlasov-Ampère equation is achieved, with a forward Semi-Lagrangian method introduced in [10]. The Vlasov equation belongs to the kinetic way of simulating plasmas evolution, and is coupled with the Poisson's equation, or equivalently under charge conservation, the Ampère's one, which self-consistently rules the electric field evolution. In order to ensure having proper physical solutions, it is necessary that the scheme preserves charge numerically. B-Spline deposition will be used for the interpolation step. The solving of the characteristics will be made with a Runge-Kutta 2 method and with a Cauchy-Kovalevsky procedure.
\end{abstract}

Keywords: Semi-Lagrangian method, Charge conservation, Runge-Kutta, Cauchy-Kovalevsky, B-spline deposition.

\section{Contents}

1 Introduction $\quad 1$

2 The continuous problem $\quad 3$

2.1 The Vlasov-Ampère and Vlasov-Poisson models . . . . . . . . . . . . . . . . . 3

2.2 The quasi-relativistic Vlasov-Maxwell model . . . . . . . . . . . . . . . . . . . 4

3 The discrete problems $\quad 5$

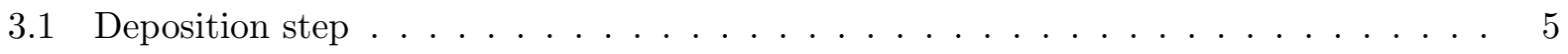

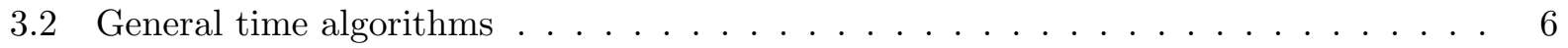

3.2 .1 Vlasov-Ampère . . . . . . . . . . . . . . . . . . . 6

3.2 .2 QR 1D Vlasov-Maxwell . . . . . . . . . . . . . . . . . 8

4 The charge preserving algorithms $\quad 9$

4.1 Solving the electric field, Ampère vs Poisson . . . . . . . . . . . . . . . . . 9

4.2 Charge conservation . . . . . . . . . . . . . . . . . 10

5 Numerical results $\quad 13$

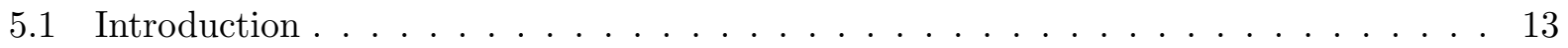

5.2 Linear Landau damping . . . . . . . . . . . . . . . . . . . . . 13

5.3 Two stream instability . . . . . . . . . . . . . . . . . 15

*INRIA-Nancy-Grand Est, CALVI Project

${ }^{\dagger}$ IRMA Strasbourg et INRIA-Nancy-Grand Est, CALVI Project 
5.4 Bump on Tail . . . . . . . . . . . . . . . . . . . . . 18

5.5 QR Vlasov-Maxwell test case . . . . . . . . . . . . . . . . . 21

5.6 Numerical Synthesis . . . . . . . . . . . . . . . . . . . . . 26

6 Conclusion $\quad 26$

\section{Contents}

\section{Introduction}

In order to describe the dynamics of charged particles in a plasma or in a propagating beam, the Vlasov equation can be used to calculate the response of the plasma to the electromagnetic fields. The unknown $f(t, x, v)$ which depends on the time $t$, the space $x$ and the velocity $v$ represents the distribution function of the studied particles. The coupling with the self-consistent electromagnetic fields is taken into account through the Maxwell's equations.

The numerical solution of such systems is most of the time performed using Particle In Cell (PIC) methods, in which the plasma is approximated by macro-particles (see [3]). They are advanced in time with the electromagnetic fields which are computed on a grid. However, despite their capability to treat complex problems, PIC methods are inherently noisy. This becomes problematic when low density or highly turbulent regions are studied. Hence, numerical methods which discretize the Vlasov equation on a grid of the phase space can offer a good alternative to PIC methods (see $[7,16,17,34,6,12,28])$.

An important issue for electromagnetic PIC or Vlasov solvers in which the fields are computed through the Maxwell's equations, is the problem of discrete charge conservation. The electric and magnetic fields via Ampère and Faraday's equations have to be computed in such a way that it satisfies a discrete Gauss law at each time step. Indeed, the charge and current densities computed from the particles (for PIC methods) or from $f$ (for Vlasov methods) do not verify the continuity equation so that the Maxwell's equations with these sources might be ill-posed.

Two main issues have been explored in the literature, mainly in the PIC context. The first one consists in modifying the inconsistent electric field resulting from an ill-posed Maxwell solver (see $[24,25])$. In the second approach, the current is computed in a specific way so as to enforce a discrete continuity equation (see $[36,1,15,35]$ ). The second class of methods has the advantage of being local and does not modify the electromagnetic field away from the source, which can generate errors in some applications.

More recently, Sircombe and Arber managed to create a 4D Vlasov-Maxwell charge preserving scheme using a split Eulerian approach (VALIS code described in [30]). They take full benefits of conservative methods computing the current using the fluxes in space after each spatial advection. In addition to some specifical properties that can be controled through filters (positivity, monotonicity), conservative methods applied to multidimensional problems can be solved through the succession of unidimensional problems thanks to a directional splitting like for instance in [27]. Thus, conservative methods have proven very efficient for the solving of transport equations (for details see $[9,16,17,38,8])$. In this work, we also deal with a phase space grid to simulate the Vlasov equation, but using the Forward semi-Lagrangian method (FSL detailed in [10]) ; as explained in [10], since FSL bears similarities with PIC methods, we shall extend the approach of $[36,1,13]$ to our FSL context. In a few words, the time-dependent current is averaged over the one-step trajectory of the particles (which correspond to the grid points in FSL).

The FSL approach has been developed on a cubic B-splines reconstruction. Different time algorithms are proposed to solve the characteristics of the Vlasov equations: Runge-Kutta or 
Cauchy-Kovaleskaya algorithms. As explained in [10] the main advantages of the FSL method are : $(i)$ it is conservative (thanks to the partition of the unity), $(i i)$ it can be easily extended to arbitrary high order time algorithms in its unsplit form (using CK algorithms), and (iii) it is equivalent to the BSL counterpart when 1D constant advections are considered.

The coupling of FSL with charge preserving algorithms is the main goal of this work. We focus on the 1D Vlasov-Ampère and quasi-relativistic Vlasov-Maxwell models to show the feasibility and the advantages of the approach. The extension to the 2D Vlasov-Maxwell case will be the object of a future work in which we hope to present an alternative approach to the VALIS one presented in [30]. Here, we will see that if a discrete charge conservation is ensured and if the Poisson's equation is satisfied initially, then the electric field computed by the Ampère's equation automatically obeys the finite difference version of the Poisson's equation to machine precision without solving it. The key point is the computation of the current. For a given time computation of the characteristics (RK, CK), a numerical integration of the time-dependent current over the affine approximation of the characteristics is done. Since cubic B-splines are used, the current is polynomial of degree 3 so that a numerical integration using a Gaussian quadrature is exact. We are then able to prove that the so-computed current: $(i)$ satisfies the continuity equation and (ii) generates (through the Ampère's equation) an electric field compatible with Poisson (without solving it). We then observe on numerical results that for a given time algorithms in FSL, the solution of the charge preserving scheme for the Vlasov-Ampère equation is equivalent to the solution of the Vlasov-Poisson equation. This is a first step towards more realistic 4D Vlasov-Maxwell simulations.

This paper is organized as follows. In the first part, the continuous problems of Vlasov-Poisson, Vlasov-Ampère and quasi-relativistic (QR) Vlasov-Maxwell 1D are presented. In the second part, the associated discrete problems and the numerical schemes to solve them are explained. Then, the charge preserving method will be detailed. Finally, numerical results will be displayed, especially a test case about 1D quasi relativistic Vlasov-Maxwell.

\section{The continuous problem}

\subsection{The Vlasov-Ampère and Vlasov-Poisson models}

Let us consider $f(t, x, v) \geq 0$ being the distribution function of electrons in phase-space, and $E(t, x)$ the self consistent electric field. We shall also consider an initially neutralizing population of immobile ions, in order to ensure the global neutrality of the plasma. Nevertheless, only the population of electrons will be taken care of in this work. The dimensionless periodical VlasovAmpère (resp. Poisson) system for this population of electrons reads:

$$
\begin{gathered}
\partial_{t} f+v \partial_{x} f+E(t, x) \partial_{v} f=0 \\
\partial_{t} E(t, x)=-J(t, x)+\bar{J}(t)=-\int_{\mathbb{R}} v f(t, x, v) d v+\frac{1}{L} \int_{0}^{L} J(t, x) d x
\end{gathered}
$$

(resp.)

$$
\partial_{x} E(t, x)=\rho(t, x)=\int_{\mathbb{R}} f(t, x, v) d v-1,
$$

where $x$ and $v$ are the phase space independent variables. Continuously, these two models are equivalent under the charge conservation condition:

$$
\partial_{t} \rho(t, x)+\partial_{x} J(t, x)=0 .
$$


A periodic plasma is considered, of period L so that $x \in[0, L], v \in \mathbb{R}, t \geq 0$. The functions $f$ and $E$ are submitted to the following conditions:

$$
\begin{gathered}
f(t, 0, v)=f(t, L, v), \quad \forall v \in \mathbb{R}, \quad t \geq 0, \quad f(t, 0, v)=f(t, L, v), \\
E(t, 0)=E(t, L) \Leftrightarrow \frac{1}{L} \int_{0}^{L} \int_{\mathbb{R}} f(t, x, v) d v d x=1, \forall t \geq 0,
\end{gathered}
$$

which translate the global neutrality of the plasma. In order to get a well-posed problem, a zeromean electrostatic condition has to be added, which corresponds to a periodic electric potential:

$$
\int_{0}^{L} E(t, x) d x=0, \quad \forall t \geq 0
$$

and an initial condition:

$$
f(0, x, v)=f_{0}(x, v), \quad \forall x \in[0, L], v \in \mathbb{R} .
$$

Assuming that the electric field is smooth enough, equations (2.1), (2.2) and (2.4) can be solved in the classical sense as follows. For the existence, the uniqueness and the regularity of the solutions of the following differential system, the reader is refered to [4].

The motion of the particles is solved through the following first order differential system

$$
\begin{aligned}
\frac{d X}{d t}(t ;(x, v), s) & =V(t ;(x, v), s), \\
\frac{d V}{d t}(t ;(x, v), s) & =E(t, X(t ;(x, v), s)),
\end{aligned}
$$

where $(X(t ;(x, v), s), V(t ;(x, v), s))$ are the characteristic curves at time $t$ which value at time $s$ was $(x, v)$. These characteristics are the solutions of $(2.7)$ at time $t$ with the initial conditions:

$$
X(s ;(x, v), s)=x, \quad V(s ;(x, v), s)=v .
$$

The solution of problem (2.1), (2.4) is given by

$$
f(t, x, v)=f_{0}(X(0 ;(x, v), t), V(0 ;(x, v), t)), \quad \forall x \in[0, L], v \in \mathbb{R}, t \geq 0 .
$$

Since $\partial(X, V) / \partial(x, v)=1$, it follows

$$
\frac{1}{L} \int_{0}^{L} \int_{\mathbb{R}} f(t, x, v) d v d x=\frac{1}{L} \int_{0}^{L} \int_{\mathbb{R}} f_{0}(x, v) d v d x=1 .
$$

According to previous considerations, an equivalent form of the periodic Vlasov-Poisson problem is to find $(f, E)$, smooth enough, periodic with respect to $x$, with period $L$, and to solve the equations $(2.2),(2.7),(2.8)$ and $(2.9)$.

Introducing the electrostatic potential $\varphi=\varphi(t, x)$ such that $E(t, x)=-\partial_{x} \varphi(t, x)$, and setting $G=G(x, y)$ the fundamental solution of the Laplacian operator in one dimension, that is $-\partial_{x}^{2} G(x, y)=\delta_{0}(x-y)$ with periodic boundary conditions, it comes

$$
E(t, x)=\int_{0}^{L} K(x, y)\left(\int_{\mathbb{R}} f(t, y, v) d v-1\right) d y,
$$

where

$$
K(x, y)=-\partial_{x} G(x, y)=\left\{\begin{array}{ccc}
y / L-1, & \text { if } & 0 \leq x<y \\
y / L, & \text { if } & y<x \leq L
\end{array}\right.
$$




\subsection{The quasi-relativistic Vlasov-Maxwell model}

This model describes the motion of the electrons in the laser-plasma interaction context and has been recently introduced in the literature by the physicists [21]. To derive such a model, the key points are the following: starting from the Vlasov-Maxwell equations in one dimension in space (called $\mathrm{x}$ ), and three dimensions in momentum, we make the assumption that the motions of interest are faster along the direction of propagation of the laser than in the associated transversal directions. Then, it is reasonable to consider that the electrons are monokinetic in the directions transversal to $\mathrm{x}$. We refer the reader to $[19,23,18,2]$ for more details. In particular, for the normalizations and the mathematical study, see [5]. After some computations (see [5]) one gets the QR 1D Vlasov-Maxwell system:

$$
\frac{\partial f}{\partial t}+v(p) \frac{\partial f}{\partial x}+\left(E_{x}-\frac{\partial\left(|A|^{2} / 2\right)}{\partial x}\right) \frac{\partial f}{\partial p}=0
$$

where $v(p)=\frac{p}{\gamma(p)}, \gamma(p)=\left(1+p^{2}\right)^{\frac{1}{2}}$ being the QR Lorentz factor, and $A=\left(0, A_{y}, A_{z}\right)$ being the vector potential. The Vlasov equation has to be coupled with the following $1 D$ Maxwell's equations:

$$
\begin{gathered}
\frac{\partial E_{y}(t, x)}{\partial t}=-\frac{\partial B_{z}(t, x)}{\partial x}+A_{y}(t, x) \rho_{\gamma}(t, x), \\
\frac{\partial E_{z}(t, x)}{\partial t}=\frac{\partial B_{y}(t, x)}{\partial x}+A_{z}(t, x) \rho_{\gamma}(t, x) .
\end{gathered}
$$

Note that $\rho_{\gamma}=\rho$ in the QR case.

$$
\begin{gathered}
\frac{\partial B_{y}(t, x)}{\partial t}=\frac{\partial E_{z}(t, x)}{\partial x} \\
\frac{\partial B_{z}(t, x)}{\partial t}=-\frac{\partial E_{y}(t, x)}{\partial x} .
\end{gathered}
$$

The components of the vector potential are then computed:

$$
\begin{aligned}
& \frac{\partial A_{y}(t, x)}{\partial t}=-E_{y}(t, x), \\
& \frac{\partial A_{z}(t, x)}{\partial t}=-E_{z}(t, x) .
\end{aligned}
$$

The longitudinal component of the electric field is obtained through the Poisson's equation:

$$
\frac{\partial E_{x}(t, x)}{\partial x}=\int_{\mathbb{R}} f(t, x, p) d p-1
$$

or equivalently under condition (2.3) through the Ampère's equation:

$$
\frac{\partial E_{x}(t, x)}{\partial t}=-\int_{\mathbb{R}} v(p) f(t, x, p) d p+\frac{1}{L} \int_{0}^{L} J(t, x) d x,=-J(t, x)+\bar{J}(t) .
$$

Initial conditions have to be added for the previous equations:

$$
\begin{gathered}
f(0, x, p)=f_{0}(x, p), \quad(x, p) \in \mathbb{R}^{2}, \\
\left(E, A, \partial_{x} A\right)(0, x)=\left(E_{0}, A_{0}, A_{1}\right)(x), \quad x \in \mathbb{R}
\end{gathered}
$$


The trajectories of the particles are solved through the following system:

$$
\begin{aligned}
\frac{d X}{d t}(t ;(x, p), s) & =v(P(t ;(x, p), s)), \\
\frac{d P}{d t}(t ;(x, p), s) & =\left(E(t, X(t ;(x, p), s))-\frac{\partial\left(|A|^{2} / 2\right)}{\partial x}(t, X(t ;(x, p), s))\right),
\end{aligned}
$$

where $(X(t ;(x, p), s), P(t ;(x, p), s))$ are the characteristic curves at time $t$ which value at time $s$ was $(x, p)$, solutions of $(2.10)$ at time $t$ with the initial conditions:

$$
X(s ;(x, p), s)=x, \quad P(s ;(x, p), s)=p .
$$

\section{The discrete problems}

In this section, the overall strategy of the FSL algorithm is presented. As detailed in [10], it is composed of two main steps: computation of the characteristics forwardly, and remapping of the distribution function on a phase space grid (deposition step).

First, the deposition step will be recalled. Then, time algorithms will be detailed for both models (1D Vlasov-Ampère and 1D Vlasov-Maxwell), i.e. how to advance the characteristics together with the update of the electromagnetic fields.

\subsection{Deposition step}

The two models have this step in common, the slight difference is that the characteristics are velocity ones for Vlasov-Ampère and impulsion ones for Vlasov-Maxwell. Thus, the deposition step shall be given for Vlasov-Ampère. Changing all $v$ and $V$ with $p$ or $P$ and solving (2.10) instead of (2.7) is merely needed to get this step for Vlasov-Maxwell.

A grid of the phase-space will be given, with $N_{x}$ and $N_{v}$ the number of points in the $x$ direction $\left[0, L_{x}\right]$ and the $v$ direction $\left[-v_{\max }, v_{\max }\right]$. We then define

$$
\Delta x=L_{x} / N_{x}, \quad \Delta v=2 v_{\max } / N_{v}, x_{i}=i \Delta x, \quad v_{j}=-v_{\max }+j \Delta v .
$$

The discrete distribution function is initialized this way:

$$
f_{i, j}^{0}=f_{0}\left(x_{i}, v_{j}\right) .
$$

Then, if the values of $f$ at time $t^{n}$ are known on the grid, the discrete distribution function between time $\left[t^{n}, t^{n+1}\right]$ is a projection onto a cubic B-splines basis:

$$
f(t, x, v)=\sum_{k, l} \omega_{k, l}^{n} S\left(x-X\left(t ; x_{k}, v_{l}, t^{n}\right)\right) S\left(v-V\left(t ; x_{k}, v_{l}, t^{n}\right)\right) .
$$

$\left(X\left(t ; x_{k}, v_{l}, t^{n}\right), V\left(t ; x_{k}, v_{l}, t^{n}\right)\right)$ are the characteristics at time $t$, solutions of the two dimensional system (2.7), which origin at time $t^{n}$ was the grid point $\left(x_{k}, v_{l}\right)$. The $\omega_{k, l}^{n}$ are the coefficients of the projection of $f^{n}$ onto the spline basis. The cubic B-spline $S$ is defined as follows

$$
6 S(x)= \begin{cases}(2-|x|)^{3} & \text { if } 1 \leq|x| \leq 2 \\ 4-6 x^{2}+3|x|^{3} & \text { if } 0 \leq|x| \leq 1 \\ 0 & \text { otherwise }\end{cases}
$$

Knowing the end of the characteristics, the distribution function is updated this way:

$$
\begin{aligned}
f\left(t^{n+1}, x_{i}, v_{j}\right) & =\sum_{k, l} \omega_{k, l}^{n} S\left(x_{i}-X\left(t^{n+1} ; x_{k}, v_{l}, t^{n}\right)\right) S\left(v_{j}-V\left(t^{n+1} ; x_{k}, y_{l}, t^{n}\right)\right), \\
& =\sum_{k, l} \omega_{k, l}^{n+1} S\left(x_{i}-x_{k}\right) S\left(y_{j}-y_{l}\right) .
\end{aligned}
$$


Adding boundary conditions (for example the value of the normal derivative of $f$ at the boundaries), a set of linear systems in each direction is obtained, from which the weights $\omega_{k, l}^{n}$ can be computed as in $[34,20]$. This phase will be called the deposition one. We detail in the next sequel two strategies to compute the characteristics numerically. Let us remark that the FSL method is conservative thanks to the partition of the unity property of B-splines. Moreover, when one-dimensional constant advections are considered, the forward approach is equivalent to the backward one, which is in turn equivalent in this case to its conservative counterpart (PSM: Parabolic Splines Method introduced in [38]). See [9] for more details about this last equivalence.

\subsection{General time algorithms}

\subsubsection{Vlasov-Ampère}

At time $t^{n} ; x^{n}, v^{n}, E^{n}, f^{n}$ are known. We first have to solve:

$$
\begin{aligned}
& \frac{d X}{d t}(t ;(x, v), s)=V(t ;(x, v), s) \\
& \frac{d V}{d t}(t ;(x, v), s)=E(t, X(t ;(x, v), s))
\end{aligned}
$$

with $\left(x_{k}, v_{l}\right)$ as initial values.

Runge-Kutta 2 To compute the end of the characteristics starting at $\left(x_{k}, v_{l}\right)$, a Runge-Kutta 2 algorithm can be applied. It reads

- Step 1: $\tilde{x}_{k, l}^{n+1}=x_{k}+\Delta t v_{l}$,

- Step 2: $\tilde{v}_{k, l}^{n+1}=v_{l}+\Delta t E\left(t^{n}, x_{k}\right)$,

- Step 3: $x_{k, l}^{n+1}=x_{k}+\frac{\Delta t}{2}\left(v_{l}+\tilde{v}_{k, l}^{n+1}\right)$,

- Step 4: $v_{k, l}^{n+1}=v_{l}+\frac{\Delta t}{2}\left(E\left(t^{n}, x_{k}\right)+E\left(t^{n+1}, \tilde{x}_{k, l}^{n+1}\right)\right)$.

In order to perform the last step of the Runge-Kutta 2 algorithm, the electric field at time $t^{n+1}$ is needed. In the classical way, $x_{k, l}^{n+1}$ is used to compute $\rho^{n+1}$, and then the Poisson's equation is solved to compute the new electric field. We will see later how $E$ is advanced for a charge preserving algorithm and the resolution of the Ampère's equation. After this step, the deposition is made.

Cauchy-Kovalevsky procedure The strategy was developed in [29] and more details can be found there. The idea is to get high order approximations of the characteristics using Taylor expansions in time. And then, using the charge conservation equation, and higher velocity moments of the Vlasov equation, it becomes possible to replace time derivatives with terms containing only spatial derivatives and moments at time $t^{n}$. All these sources can be easily computed. Up to third order these Taylor expansions in time lead to:

$$
\begin{gathered}
X^{n+1}=X^{n}+\Delta t V^{n}+\frac{\Delta t^{2}}{2} E^{n}\left(X^{n}\right)+\frac{\Delta t^{3}}{6} \frac{d}{d t} E(X(t), t)_{\mid t=t^{n}}, \\
V^{n+1}=V^{n}+\Delta t E^{n}\left(X^{n}\right)+\frac{\Delta t^{2}}{2} \frac{d}{d t} E(X(t), t)_{\mid t=t^{n}}+\frac{\Delta t^{3}}{6} \frac{d^{2}}{d t^{2}} E(X(t), t)_{\mid t=t^{n}} .
\end{gathered}
$$


In order to be able to compute all the terms of these expansions the two first total time derivatives of $E(X(t), t)$ are needed. The first one is

$$
\begin{aligned}
\frac{d}{d t} E(X(t), t) & =\frac{\partial E}{\partial t}(X(t), t)+\frac{d X}{d t}(t) \frac{\partial E}{\partial x}(X(t), t), \\
& =-J(X(t), t)+\bar{J}(t)+V(t) \rho(X(t), t),
\end{aligned}
$$

where $\rho(x, t)=\int f(x, v, t) d v-1, J(x, t)=\int f(x, v, t) v d v$ and $\bar{J}(t)=1 / L \int_{0}^{L} J(x, t) d x$. Indeed, the Poisson's equation yields $\partial_{x} E=\rho$ and integrating the Vlasov equation with respect to velocity, yields the charge conservation equation $\partial_{t} \rho+\partial_{x} J=0$. Hence taking the derivative of the Poisson's equation with respect to time and using this equation we get

$$
\partial_{x}\left(\partial_{t} E+J\right)=0
$$

From which is obtained, as $\int_{0}^{L} E(x, t) d x=0$ :

$$
\partial_{t} E=-J+\bar{J}
$$

The second total time derivative of $E(X(t), t)$ writes

$$
\begin{aligned}
\frac{d^{2}}{d t^{2}} E(X(t), t) & =-\partial_{t} J(X(t), t)-V(t) \partial_{x} J(X(t), t)+\frac{d \bar{J}}{d t}(t), \\
& +E(X(t), t) \rho(X(t), t)+V(t)\left(\partial_{t} \rho(X(t), t)+V(t) \partial_{x} \rho(X(t), t)\right) .
\end{aligned}
$$

To get rid of time derivatives, the strategy is to replace them with space derivatives. Concerning $\rho$, the charge conservation equation $(2.3)$ is used:

$$
\partial_{t} \rho(X(t), t)=-\partial_{x} J(X(t), t) .
$$

In order to compute the time derivative of the current $J$ in terms of spatial derivatives, the Vlasov equation (2.1) is used, multiplied with $v$, and integrated with respect to $v$. This leads to

$$
\partial_{t} J+\partial_{x} I_{2}+E \int_{\mathbb{R}} \partial_{v} f v d v=0
$$

where $I_{n}(x, t)=\int_{\mathbb{R}} f(x, v, t) v^{n} d v$. Hence, since $f$ vanishes at infinity an integration by parts enables to get

$$
\partial_{t} J(X(t), t)=-\partial_{x} I_{2}(X(t), t)+E(X(t), t)(1+\rho(X(t), t)) .
$$

Moreover, the conservation of impulsion is respected in this model, so that:

$$
\frac{d \bar{J}}{d t}(t)=0 \text {. }
$$

In conclusion, a $3^{\text {rd }}$ order Cauchy-Kovalevsky (CK3) time algorithm can be derived, following (2.3), (3.13), (3.14):

$$
X^{n+1}=X^{n}+\Delta t V^{n}+\frac{\Delta t^{2}}{2} E^{n}\left(X^{n}\right)+\frac{\Delta t^{3}}{6} \varphi^{n}\left(X^{n}, V^{n}\right) .
$$

and

$$
V^{n+1}=V^{n}+\Delta t E^{n}\left(X^{n}\right)+\frac{\Delta t^{2}}{2} \varphi^{n}\left(X^{n}, V^{n}\right)+\frac{\Delta t^{3}}{6} \phi^{n}\left(X^{n}, V^{n}\right) .
$$

where

$$
\begin{gathered}
\varphi^{n}\left(X^{n}, V^{n}\right)=V^{n} \rho^{n}\left(X^{n}\right)-J^{n}\left(X^{n}\right)+\bar{J} \\
\phi^{n}\left(X^{n}, V^{n}\right)=\partial_{x} I_{2}\left(X^{n}, t^{n}\right)-E^{n}\left(X^{n}\right)-2 V^{n} \partial_{x} J\left(X^{n}, t^{n}\right)+\left(V^{n}\right)^{2} \partial_{x} \rho\left(X^{n}, t^{n}\right) .
\end{gathered}
$$


Remark 3.1 Obviously, in order to get a second order algorithm (CK2), you just keep the terms until $\Delta t^{2}$ included.

With CK procedures, the characteristics can be solved immediately at the beginning of the time step, since everything needed is at once available. Once you know $x^{n+1}$, you can compute $\rho^{n+1}$ and compute $E^{n+1}$ like in RK with the Poisson's equation.

\subsubsection{QR 1D Vlasov-Maxwell}

Suppose that at time $t^{n}: f^{n}, E_{x}^{n}, B_{y, z}^{n}, A_{y, z}^{n}, E_{y, z}^{n-\frac{1}{2}}, x^{n}, p^{n}$ are known. A Yee algorithm (see [11]) will be used for the solving of the electromagnetic fields. The general time algorithm writes:

- Advance electric field $E_{y, z}$ on a time step:

$$
\begin{aligned}
& E_{y, i}^{n+\frac{1}{2}}=E_{y, i}^{n-\frac{1}{2}}-\frac{\Delta t}{\Delta x}\left(B_{z, i+\frac{1}{2}}^{n}-B_{z, i-\frac{1}{2}}^{n}\right)+\Delta t A_{y, i}^{n} \rho_{\gamma, i}^{n}, \\
& E_{z, i}^{n+\frac{1}{2}}=E_{z, i}^{n-\frac{1}{2}}+\frac{\Delta t}{\Delta x}\left(B_{y, i+\frac{1}{2}}^{n}-B_{y, i-\frac{1}{2}}^{n}\right)+\Delta t A_{z, i}^{n} \rho_{\gamma, i}^{n} .
\end{aligned}
$$

- Advance the magnetic field $B_{y, z}^{n}$ on a time step:

$$
\begin{aligned}
& B_{y, i-\frac{1}{2}}^{n+1}=B_{y, i-\frac{1}{2}}^{n}+\frac{\Delta t}{\Delta x}\left(E_{z, i}^{n+\frac{1}{2}}-E_{z, i-1}^{n+\frac{1}{2}}\right), \\
& B_{z, i-\frac{1}{2}}^{n+1}=B_{z, i-\frac{1}{2}}^{n}-\frac{\Delta t}{\Delta x}\left(E_{y, i}^{n+\frac{1}{2}}-E_{y, i-1}^{n+\frac{1}{2}}\right) .
\end{aligned}
$$

- Advance the vector potential $A_{y, z}^{n}$ on a time step:

$$
\begin{aligned}
& A_{y, i}^{n+1}=A_{y, i}^{n}-\Delta t E_{y, i}^{n+\frac{1}{2}}, \\
& A_{z, i}^{n+1}=A_{z, i}^{n}-\Delta t E_{z, i}^{n+\frac{1}{2}} .
\end{aligned}
$$

- First step of RK2 algorithm for the characteristics:

$$
\begin{gathered}
\tilde{x}_{k, l}^{n+1}=x_{k}+\Delta t v\left(p_{l}\right), \\
\tilde{p}_{k, l}^{n+1}=p_{l}+\Delta t\left(E_{x, k}^{n}+F^{n}\left(x_{k}\right)\right), \\
x_{k, l}^{n+1}=x_{k}+\frac{\Delta t}{2}\left(v\left(p_{l}\right)+v\left(\tilde{p}_{k, l}^{n+1}\right)\right) .
\end{gathered}
$$

- Compute $E_{x}^{n+1}$ on a time step using the Poisson's equation.

- End of RK2 algorithm

$$
p_{k, l}^{n+1}=p_{l}+\frac{\Delta t}{2}\left(E^{n}\left(x_{k}\right)+F^{n}\left(x_{k}\right)+E^{n+1}\left(x_{k, l}^{n+1}\right)+F^{n+1}\left(x_{k, l}^{n+1}\right)\right) .
$$

- Deposition,

where $F(t, x)=-A_{y}(t, x) B_{z}(t, x)+A_{z}(t, x) B_{y}(t, x)$. 


\section{The charge preserving algorithms}

In the previous section, it was seen that the electric field has to be updated thanks to the Poisson's equation. In this section, we are going to explain how to update $E$ solving the Ampère's equation without solving the Poisson's one, but ensuring it is satisfied thanks to charge preserving algorithms.

\subsection{Solving the electric field, Ampère vs Poisson}

Let us precise that for all methods, the field will be computed on the dual mesh $\left(x_{i+\frac{1}{2}}\right)_{i \in \mathrm{N}}$ on which $\left(E\left(t^{n}, x_{i+\frac{1}{2}}\right)\right)$ is supposed to be known. In order to get $\left(E\left(t^{n}, x_{i}\right)\right)$, linear or cubic spline interpolation is used. The key is how $E^{n+1}$ is computed. Let us begin with the Ampère's equation. Up to second order accuracy in time, its approximation reads:

$$
E\left(t^{n+1}, x_{i+\frac{1}{2}}\right)=E\left(t^{n}, x_{i+\frac{1}{2}}\right)-\Delta t\left(J_{i+\frac{1}{2}}^{n+\frac{1}{2}}+\bar{J}\right) .
$$

The way $J$ is calculated will be explained later. The considered finite difference discrete approximation of the Poisson's equation reads:

$$
E\left(t^{n+1}, x_{i+\frac{1}{2}}\right)=E\left(t^{n+1}, x_{i-\frac{1}{2}}\right)+\Delta x \rho_{i}^{n+1} .
$$

Let us prove now that if the discrete charge conservation is enforced, the electric fields computed with Poisson or Ampère are equal.

Proposition 1 The two methods will be actually the same if the charge and current densities verify the discrete charge conservation equation of second order derived from a Yee algorithm:

$$
\frac{\rho_{i}^{n+1}-\rho_{i}^{n}}{\Delta t}+\frac{J_{i+\frac{1}{2}}^{n+\frac{1}{2}}-J_{i-\frac{1}{2}}^{n+\frac{1}{2}}}{\Delta x}=0 .
$$

Proof: Let us prove that if Poisson is true at time $t^{n}$, and discrete charge conservation (4.17) is verified, $E$ with Poisson and Ampère at time $t^{n+1}$ will be equal. Using the Ampère's equation and (4.17)

$$
\begin{aligned}
E^{n+1}\left(x_{i+\frac{1}{2}}\right)-E^{n}\left(x_{i+\frac{1}{2}}\right) & =-\Delta t\left(J_{i+\frac{1}{2}}^{n+\frac{1}{2}}+\bar{J}\right), \\
\frac{\rho_{i}^{n+1}-\rho_{i}^{n}}{\Delta t}+\frac{J_{i+\frac{1}{2}}^{n+\frac{1}{2}}-J_{i-\frac{1}{2}}^{n+\frac{1}{2}}}{\Delta x} & =0,
\end{aligned}
$$

so that

$$
\begin{aligned}
-\Delta t\left(J_{i+\frac{1}{2}}^{n+\frac{1}{2}}-J_{i-\frac{1}{2}}^{n+\frac{1}{2}}\right) & =E^{n+1}\left(x_{i+\frac{1}{2}}\right)-E^{n}\left(x_{i+\frac{1}{2}}\right)-E^{n+1}\left(x_{i-\frac{1}{2}}\right)+E^{n}\left(x_{i-\frac{1}{2}}\right), \\
& =\Delta x\left(\rho_{i}^{n+1}-\rho_{i}^{n}\right) .
\end{aligned}
$$

Hence, if Poisson is satisfied at time $t^{n}$, i.e.

$$
E^{n}\left(x_{i+\frac{1}{2}}\right)-E^{n}\left(x_{i-\frac{1}{2}}\right)=\Delta x \rho_{i}^{n},
$$

Poisson is automatically satisfied at time $t^{n+1}$,

$$
E^{n+1}\left(x_{i+\frac{1}{2}}\right)-E^{n+1}\left(x_{i-\frac{1}{2}}\right)=\Delta x \rho_{i}^{n+1},
$$

which is what was required. 


\subsection{Charge conservation}

As it was proved in the last subsection, if $E$ is initialized through Poisson, the problem can be solved on each time step with Ampère, without solving the Poisson's equation if the charge and current densities satisfy (4.17). Now a way to compute compatible $\rho$ and $J$ is proposed:

Compute $\rho$ : Let us explain first how $\rho$ is computed: Once $\left(x_{k, l}^{n+1}\right)$ is known, it is possible to compute $\rho$ on the mesh using a deposition:

$$
\rho\left(t^{n+1}, x_{i}\right)=\sum_{k, l} \omega_{k, l}^{n} S\left(x_{i}-X\left(t^{n+1} ;\left(x_{k}, v_{l}\right), t^{n}\right)\right)-1 .
$$

Then, (4.16) is solved and $E\left(t^{n+1}, x_{i+\frac{1}{2}}\right)$ can be computed for each $i$, adding the zero-mean condition $(2.5)$.

Compute $J$ - Discrete charge conservation: The discrete charge conservation equation will be used in order to find $J$; following Villasenor Buneman and Barthelmé [36, 1]:

$$
\begin{aligned}
\frac{\rho_{i}^{n+1}-\rho_{i}^{n}}{\Delta t} & =\frac{1}{\Delta t} \int_{t^{n}}^{t^{n+1}} \partial_{t} \rho\left(x_{i}, t\right) \\
& =\frac{1}{\Delta t} \sum_{k, l} \omega_{k, l}^{n} \int_{t^{n}}^{t^{n+1}} \frac{d}{d t} S^{3}\left(x_{i}-X_{k, l}(t)\right) d t \\
& =-\frac{1}{\Delta t} \sum_{k, l} \omega_{k, l}^{n} \int_{t^{n}}^{t^{n+1}} \frac{d X_{k, l}(t)}{d t} S^{3^{\prime}}\left(x_{i}-X_{i, j}(t)\right) d t \\
& =-\frac{1}{\Delta t \Delta x} \sum_{k, l} \omega_{k, l}^{n} \int_{t^{n}}^{t^{n+1}} \frac{d X_{k, l}(t)}{d t}\left(S^{2}\left(x_{i+\frac{1}{2}}-X_{k, l}(t)\right)-S^{2}\left(x_{i-\frac{1}{2}}-X_{k, l}(t)\right)\right) d t, \\
& =-\left(\frac{J_{i+\frac{1}{2}}^{n+\frac{1}{2}}-J_{i-\frac{1}{2}}^{n+\frac{1}{2}}}{\Delta x}\right)
\end{aligned}
$$

where $X_{k, l}(t)=X\left(t ;\left(x_{k}, v_{l}\right), t^{n}\right)$, and $S^{2}$ the quadratic B-spline. $S^{2}$ and $S^{3}$ are linked using:

$$
\frac{d S^{3}(x)}{d x}=S^{2}\left(x+\frac{1}{2}\right)-S^{2}\left(x-\frac{1}{2}\right) .
$$

Let us remark that this update of $\rho$ depends on the time derivative of the characteristic curves. This is linked with the algorithm used to solve them. In the following paragraphs, we present for various time algorithms (CRK for charge Runge-Kutta, CCK for charge Cauchy-Kovalevsky) the way to compute J.

Runge-Kutta for Vlasov-Ampère: CRK2: What has to be understood is that the only things that matter in the particle trajectory are the beginning and the ending point of it. Then, you can derive one method for each way of approaching the trajectory with the proper beginning and ending points. For the sake of simplicity, the characteristics will be linearly approached. In that purpose, let us set:

$$
X_{k, l}(t)=x_{k}+\frac{t-t^{n}}{2}\left(v_{l}+\tilde{v}_{k, l}^{n+1}\right)
$$

so that

$$
\frac{d X_{k, l}(t)}{d t}=\frac{1}{2}\left(v_{l}+\tilde{v}_{k, l}^{n+1}\right)
$$


Thus,

$$
J_{i+\frac{1}{2}}^{n+\frac{1}{2}}=\frac{1}{2 \Delta t} \sum_{k, l} \omega_{k, l}^{n}\left(v_{l}+\tilde{v}_{k, l}^{n+1}\right) \int_{t^{n}}^{t^{n+1}} S^{2}\left(x_{i+\frac{1}{2}}-X_{k, l}(t)\right) d t .
$$

The integral in (4.18) has to be computed exactly. Supposing a CFL condition $v_{\max } \Delta t \leq \Delta x$, the particle cannot get through more than one cell, so that the integrated function is a polynom of degree 2. Thus, since the Gauss' formula with two points remains exact on polynoms till degree 3:

$$
\begin{aligned}
& \int_{t^{n}}^{t^{n+1}} S^{2}\left(x_{i+\frac{1}{2}}-X_{k, l}(t)\right) d t=\frac{\Delta t}{2} \int_{-1}^{1} S^{2}\left(x_{i+\frac{1}{2}}-X_{k, l}\left(\frac{\Delta t}{2} u+t^{n+\frac{1}{2}}\right)\right) d u \\
& =\frac{\Delta t}{2}\left(S^{2}\left(x_{i+\frac{1}{2}}-X_{k, l}\left(t^{n+\frac{1}{2}}+\frac{\Delta t}{2 \sqrt{3}}\right)\right)+S^{2}\left(x_{i+\frac{1}{2}}-X_{k, l}\left(t^{n+\frac{1}{2}}-\frac{\Delta t}{2 \sqrt{3}}\right)\right)\right) .
\end{aligned}
$$

To conclude, setting:

$J_{i+\frac{1}{2}}^{n+\frac{1}{2}}=\frac{1}{4} \sum_{k, l} \omega_{k, l}^{n}\left(v_{l}+\tilde{v}_{k, l}^{n+1}\right)\left(S^{2}\left(x_{i+\frac{1}{2}}-X_{k, l}\left(t^{n+\frac{1}{2}}+\frac{\Delta t}{2 \sqrt{3}}\right)\right)+S^{2}\left(x_{i+\frac{1}{2}}-X_{k, l}\left(t^{n+\frac{1}{2}}-\frac{\Delta t}{2 \sqrt{3}}\right)\right)\right)$.

allows to preserve charge.

Cauchy-Kovalevsky for Vlasov-Ampère: CCK:

In order to be able to follow high order algorithms easily, a linear approximation of the characteristic curve will also be applied, following (3.15):

$$
X_{k, l}(t)=x_{k}+\left(t-t^{n}\right) v_{l}+\Delta t\left(t-t^{n}\right) E^{n}\left(x_{k}\right)+\frac{\Delta t^{2}}{2}\left(t-t^{n}\right) \varphi^{n}\left(x_{k}, v_{l}\right),
$$

so that

$$
\frac{d X_{k, l}(t)}{d t}=v_{l}+\Delta t E^{n}\left(x_{k}\right)+\Delta t^{2} \varphi^{n}\left(x_{k}, v_{l}\right):=D_{k, l}^{n}
$$

Thus,

$$
J_{i+\frac{1}{2}}^{n+\frac{1}{2}}=\frac{1}{\Delta t} \sum_{k, l} \omega_{k, l}^{n} D_{k, l}^{n} \int_{t^{n}}^{t^{n+1}} S^{2}\left(x_{i+\frac{1}{2}}-X_{k, l}(t)\right) d t
$$

Using the same exact computation of the integral thanks to the Gauss' points leads to:

$$
J_{i+\frac{1}{2}}^{n+\frac{1}{2}}=\frac{1}{2} \sum_{k, l} \omega_{k, l}^{n} D_{k, l}^{n}\left(S^{2}\left(x_{i+\frac{1}{2}}-X_{k, l}\left(t^{n+\frac{1}{2}}+\frac{\Delta t}{2 \sqrt{3}}\right)\right)+S^{2}\left(x_{i+\frac{1}{2}}-X_{k, l}\left(t^{n+\frac{1}{2}}-\frac{\Delta t}{2 \sqrt{3}}\right)\right)\right) .
$$

Runge-Kutta 2 for Vlasov-Maxwell

This algorithm is the same as Runge-Kutta 2 for Vlasov-Ampère, changing $v_{l}$ and $\tilde{v}_{k, l}^{n+1}$ in $v\left(p_{l}\right)$ and $v\left(\tilde{p}_{k, l}^{n+1}\right)$. Therefore, the current which leads to a charge preserving algorithm writes:

$J_{i+\frac{1}{2}}^{n+\frac{1}{2}}=\frac{1}{4} \sum_{k, l} \omega_{k, l}^{n}\left(v\left(p_{l}\right)+v\left(\tilde{p}_{k, l}^{n+1}\right)\right)\left(S^{2}\left(x_{i+\frac{1}{2}}-X_{k, l}\left(t^{n+\frac{1}{2}}+\frac{\Delta t}{2 \sqrt{3}}\right)\right)+S^{2}\left(x_{i+\frac{1}{2}}-X_{k, l}\left(t^{n+\frac{1}{2}}-\frac{\Delta t}{2 \sqrt{3}}\right)\right)\right)$.

\section{Summary:}

Supposing $x^{n+1}$ known, $\rho$ is computed thanks to a deposition, then $J$ is computed with the previous expressions. There, $E$ can be advanced thanks to the Ampère's equation, and automatically obeys the Poisson's one. 
Remark 4.1 Note that the previous algorithms can be extended to the $4 D$ case, using the following splitting strategy:

- Solve $\partial_{t} f+v_{x} \partial_{x} f+E_{x} \partial_{v_{x}} f=0$ using one of the previous charge preserving algorithm.

- Solve $\partial_{t} f+v_{y} \partial_{y} f+E_{y} \partial_{v_{y}} f=0$ using one of the previous charge preserving algorithm.

- Solve $\partial_{t} f+v_{y} B_{z} \partial_{v_{x}} f-v_{x} B_{z} \partial_{v_{y}} f=0$, using a Boris scheme.

Let us also remark, that no prediction will be needed using a Yee algorithm for the solving of the Maxwell's equations, so reasonable CPU time can be hoped compared to standard algorithms.

\section{$5 \quad$ Numerical results}

\subsection{Introduction}

We will test our algorithms with the classical test cases already used in [10]. Let us give a few details about the following diagnostics. First, concerning the CFL condition, it will be said that $\mathrm{CFL}<1$ when $v_{\max } \Delta t<\Delta x$ and CFL $>1$ when $v_{\max } \Delta t>\Delta x$. What we call charge conservation will be the difference between $\rho$ computed with the distribution function and $\rho$ computed thanks to $J$ and the charge conservation equation:

$$
c c^{n+1}(i):=\int_{\mathbb{R}} f^{n+1}\left(x_{i}, v\right) d v-\left(\rho^{n}\left(x_{i}\right)+1-\frac{\Delta t}{\Delta x}\left(J_{i+\frac{1}{2}}^{n+\frac{1}{2}}-J_{i-\frac{1}{2}}^{n+\frac{1}{2}}\right)\right) .
$$

Finally what is called Ampère Poisson equivalence is the difference between the electric field computed with Ampère and Poisson after 1000 iterations:

$$
a p^{n+1}(i):=\frac{E^{n+1}\left(x_{i+\frac{1}{2}}\right)-E^{n+1}\left(x_{i-\frac{1}{2}}\right)}{\Delta x}+\left(\rho^{n}\left(x_{i}\right)+1-\frac{\Delta t}{\Delta x}\left(J_{i+\frac{1}{2}}^{n+\frac{1}{2}}-J_{i-\frac{1}{2}}^{n+\frac{1}{2}}\right)\right) .
$$

The classical Runge-Kutta 2 (resp Cauchy-Kovalevsky 2 and 3) algorithms, developed in section 3.2 , coupled with a resolution of the electric field with Poisson will be called RK2 (resp CK2 and CK3). The RK2 and the Cauchy-Kovalevsky algorithms with charge conservation, developed in section 4, will be denoted respectively CRK2, CCK2 and CCK3.

\subsection{Linear Landau damping}

The initial condition associated to the scaled Vlasov-Poisson equation has the following form

$$
f_{0}(x, v)=\frac{1}{\sqrt{2 \pi}} \exp \left(-v^{2} / 2\right)(1+\alpha \cos (k x)), \quad(x, v) \in[0,2 \pi / k] \times \mathbb{R},
$$

where $k=0.5$ is the wave number and $\alpha=0.001$ is the amplitude of the perturbation, so that linear regimes are considered here. A cartesian mesh is used to represent the phase space with a computational domain $[0,2 \pi / k] \times\left[-v_{\max }, v_{\max }\right], v_{\max }=6$. The number of mesh points in the spatial and velocity directions is designated by $N_{x}=64$ and $N_{v}=64$ respectively. For the charge conservation algorithms, $\Delta t=0.03$ so that the CFL condition is satisfied and $\Delta t=0.1$ are considered (the CFL is violated in this latter case).

On Fig. 1, the implemented solution of the $L^{2}$ norm of the electric field: $1 / 2\|E(t)\|_{L^{2}}^{2}$ with the Runge-Kutta algorithm without charge conservation presented in section 3.2, and the Runge-Kutta algorithm with charge conservation presented in sections 3.2 and 4 with CFL $<1$ are displayed in $\log$ scale. It can be observed that the two curves are nearly the same. Moreover, the theoretical 
damping associated to $k=0.5$, which is -0.1533 is added in order to ensure that the solution corresponds to the predicted damping. On the same figure is plotted the same result with a CFL condition superior than 1 for RK2 and CRK2, which proves that the problem comes from the violation of the CFL condition in the charge algorithm. In particular, we also plot on Fig. 2 and on Fig. 3 the charge conservation and the Ampère-Poisson equivalence with on the left $\mathrm{CFL}<1$, and on the right CFL $>1$. These results are issued from the RK algorithm and are given after 1000 iterations.

For the CK algorithm, we just plot on Fig. 4 on the left the electric energy and the theoretical damping for CK2 presented in section 3.2 and CCK2 presented in section 4, with CFL $<1$, and the same on the right for CK3 and CCK3 developed in the same sections. The curves are overlayed, as it was expected.
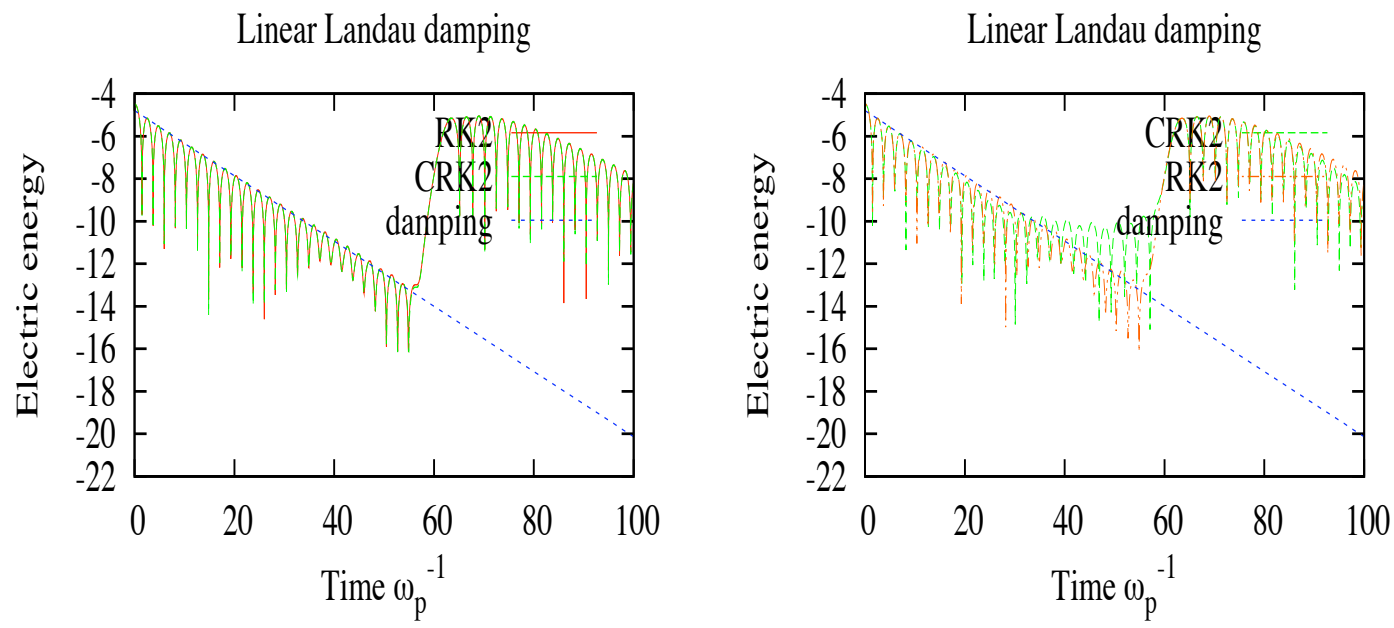

Figure 1: Linear Landau damping for $k=0.5 N_{x}=N_{v}=64, \Delta t=0.03$ (left); $\Delta t=0.1$ (right).
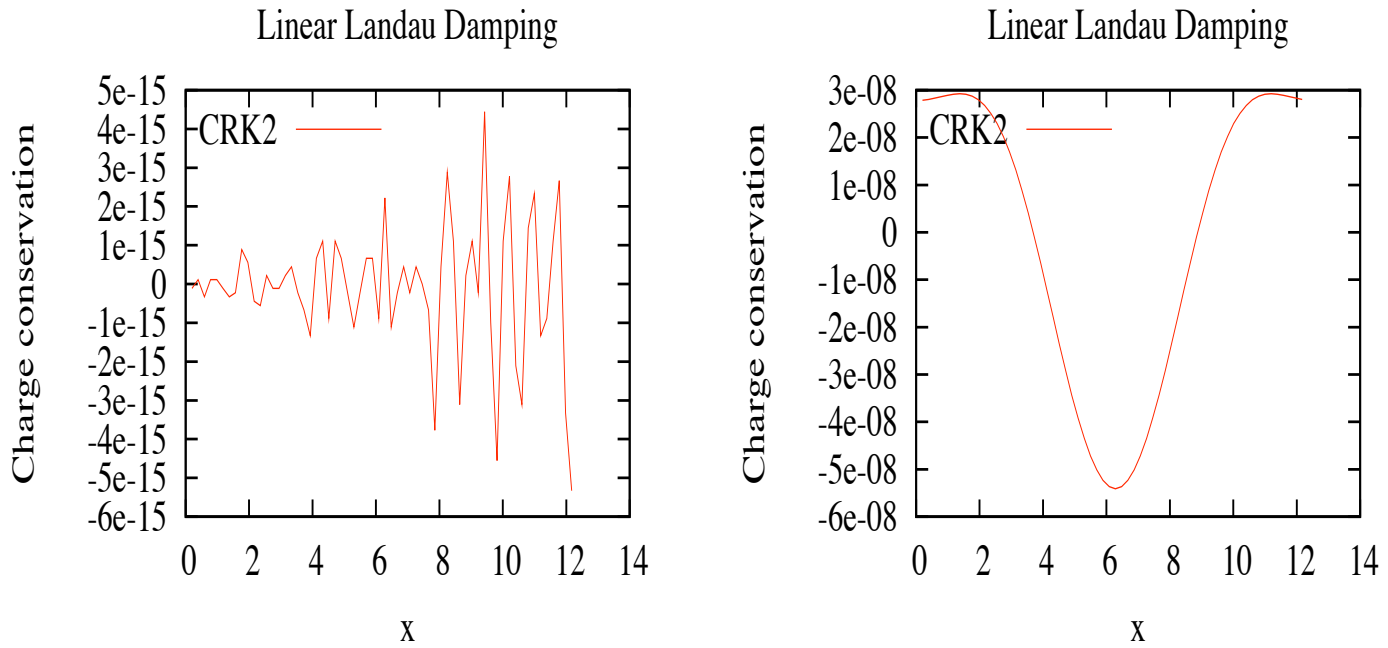

Figure 2: Charge Conservation $N_{x}=N_{v}=64, \Delta t=0.03$ (left); $\Delta t=0.1$ (right), 1000 iterations. 

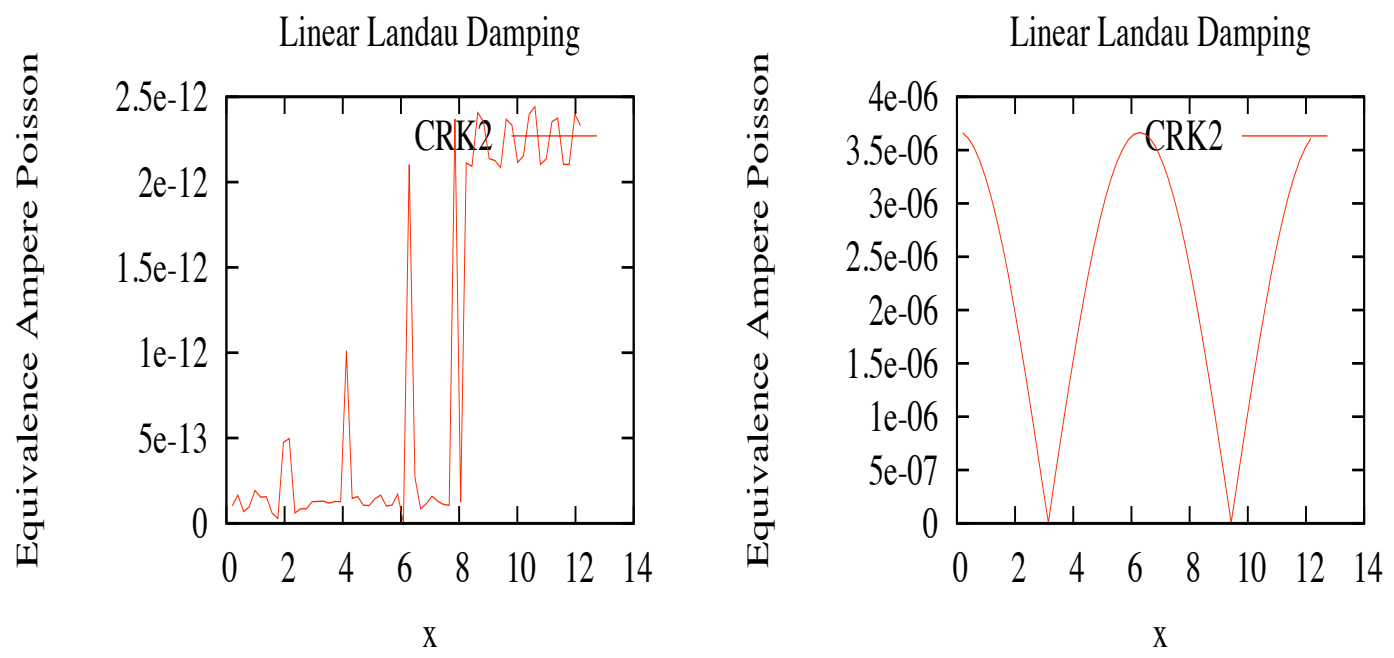

Figure 3: Ampère-Poisson equivalence, $N_{x}=N_{v}=64, \Delta t=0.03$ (left); $\Delta t=0.1$ (right), 1000 iterations.
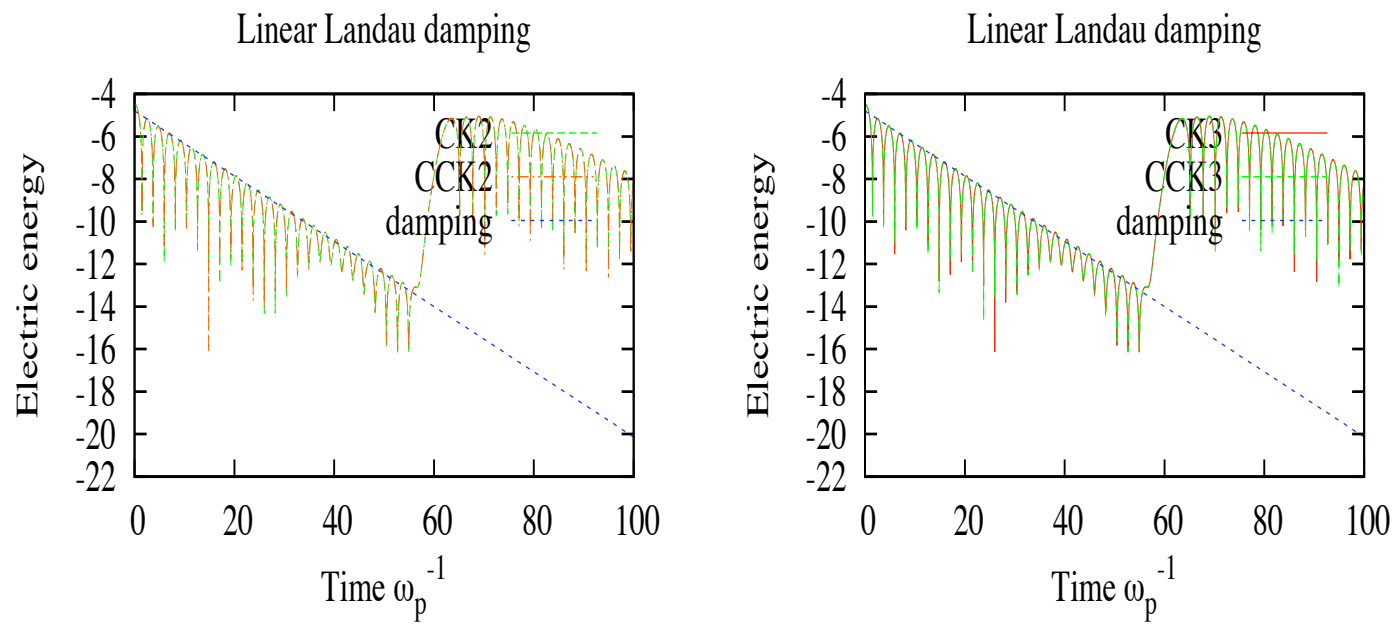

Figure 4: Electric energy for CK2 (left) and CK3 (right) $N_{x}=N_{v}=64, \Delta t=0.03$.

\subsection{Two stream instability}

This test case simulates two beams with opposite velocities that encounter (see $[17,26]$ ). The corresponding initial condition can be given by

$$
f_{0}(x, v)=\mathcal{M}(v) v^{2}[1-\alpha \cos (k x)], \quad \mathcal{M}(v)=\frac{1}{\sqrt{2 \pi}} \exp \left(-\frac{v^{2}}{2}\right),
$$

with $k=0.5$ and $\alpha=0.05$. The computational domain is $[0,2 \pi / k] \times[-9,9]$ which is sampled by $N_{x}=N_{v}=128$ points. For the charge conservation algorithms developed in section 4, CFL imposes $\Delta t<0.0109$, so the case $\mathrm{CFL}<1$ will be made with $\Delta t=0.01$, and CFL $>1$ with $\Delta t=0.1$. We are interested in the electric energy $1 / 2\|E(t)\|_{L^{2}}^{2}$, which will be displayed in log scale. The same quantities as for the previous test cases are plotted on Fig. 5, 6, 7 and 8 . The 
results are really precise for RK and CK, even in long time, under the assumption CFL $<1$ with the charge algorithms presented in section 4 . The results show a poor precision for the case CFL $>1$. On Fig. 9, the $L^{2}$ norm of the solution and the first moment in $v$ are displayed for $\Delta t=0.1$. The $L^{2}$ norm cannot be exactly conserved by any scheme using a phase space grid as soon as the grid does not resolve anymore the filaments, but the developed algorithms perform well, and have similar precision. Differences should appear taking bigger time steps, as it will be seen in the next test case. The first moment is exactly conserved for CK, which has been proved theoretically in [29]. For RK2, there is a slight decrease of the first moment, and for RK3 a small increase which remains acceptable.
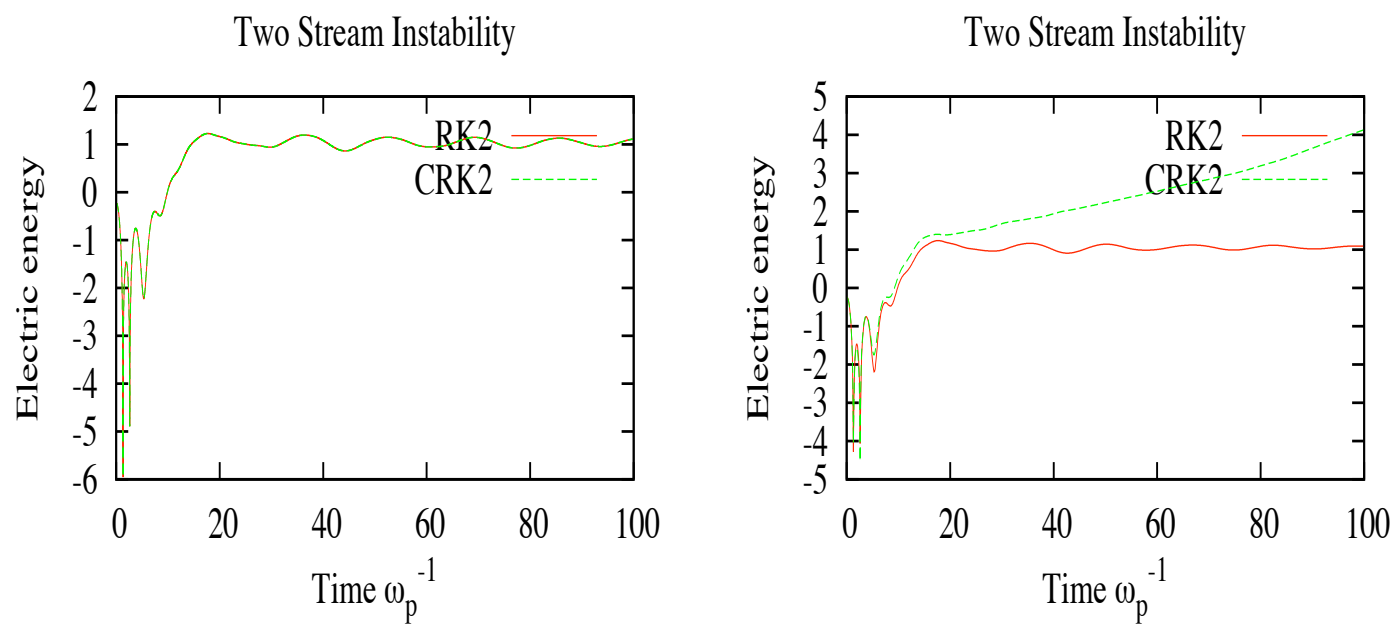

Figure 5: Electric energy: $N_{x}=N_{v}=128, \Delta t=0.01$ (left), $\Delta t=0.1$ (right).

Two stream instability

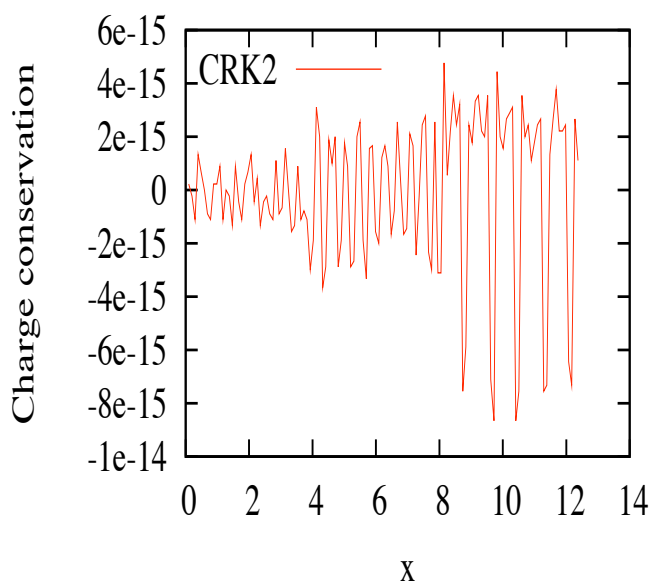

Two Stream Instability

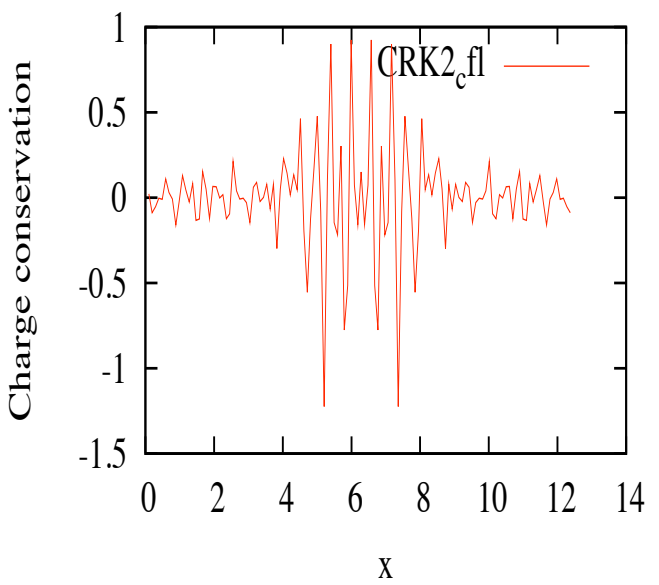

Figure 6: Charge conservation: $N_{x}=N_{v}=128, \Delta t=0.01$ (left), $\Delta t=0.1$ (right), 1000 iterations. 

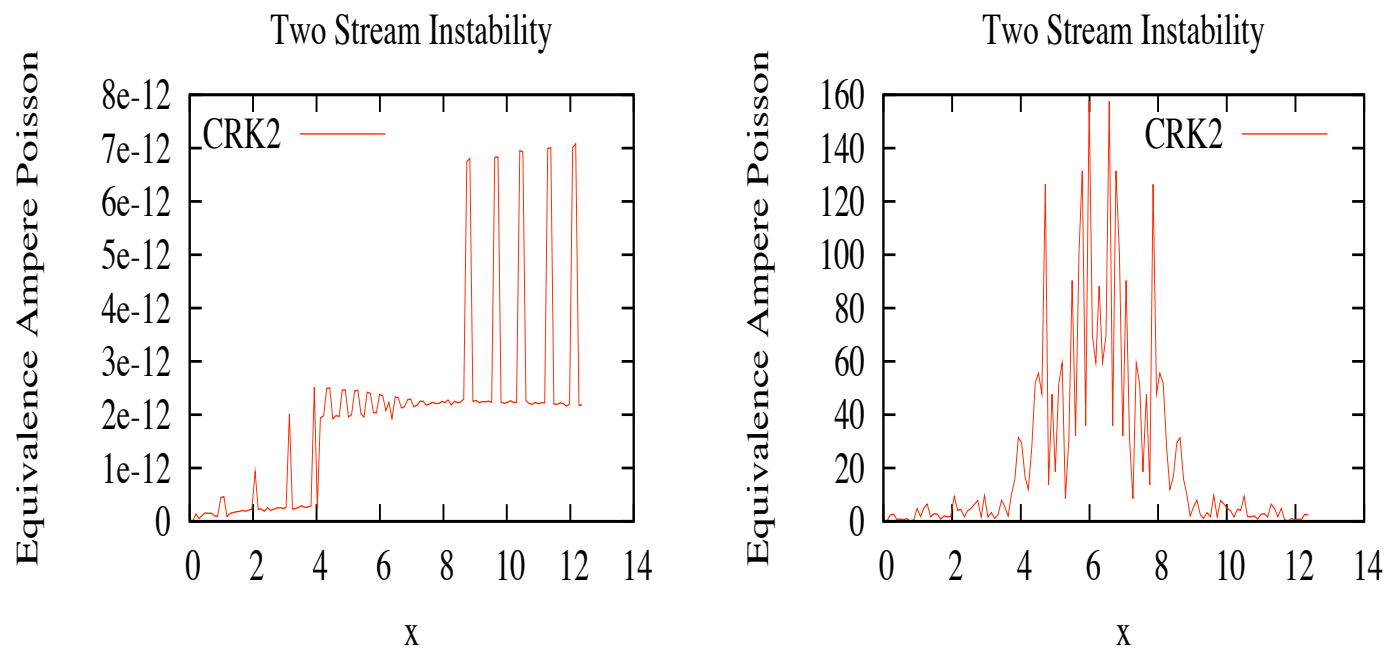

Figure 7: Ampère-Poisson equivalence, $N_{x}=N_{v}=128, \Delta t=0.01$ (left), $\Delta t=0.1$ (right), 1000 iterations.
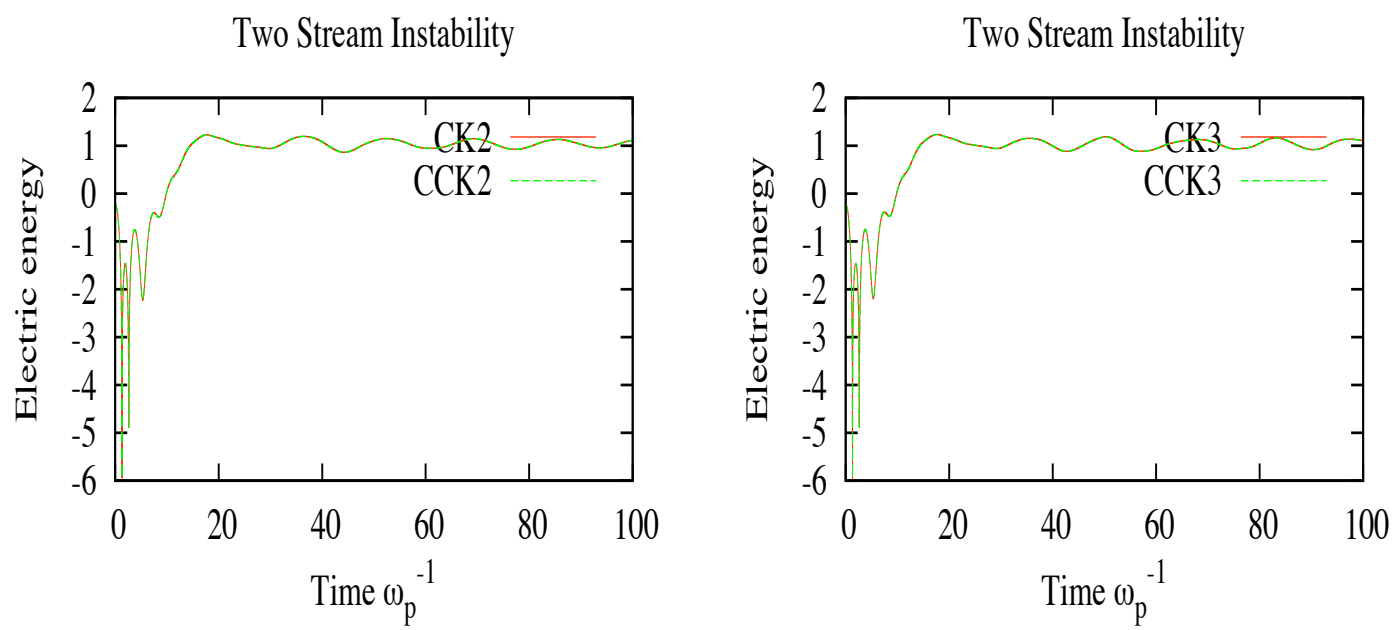

Figure 8: Electric energy CK2 (left) CK3 (right) $N_{x}=N_{v}=128, \Delta t=0.01$. 

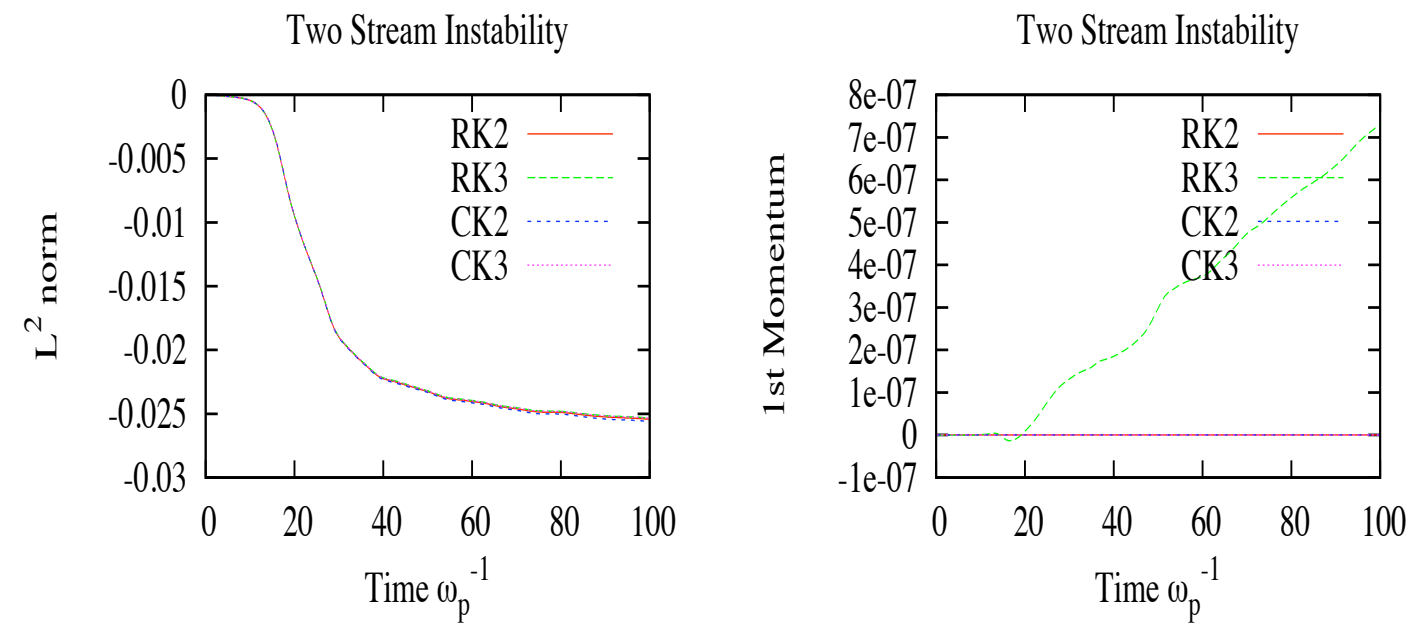

Figure 9: $L^{2}$ norm (left), First moment in v (right) $\Delta t=0.1$.

\subsection{Bump on Tail}

Next, we can apply the scheme to the bump-on-tail instability test case for which the initial condition writes (see [32])

$$
f_{0}(x, v)=\tilde{f}(v)[1+\alpha \cos (k x)]
$$

with $\alpha=0.04, k=0.3$ and

$$
\tilde{f}(v)=\frac{n_{p}}{\sqrt{2 \pi}} \exp \left(-v^{2} / 2\right)+\frac{n_{b}}{\sqrt{2 \pi}} \exp \left(-\frac{|v-u|^{2}}{2 v_{t}^{2}}\right),
$$

on the interval $[0,20 \pi]$, with periodic conditions in space. The initial condition $f_{0}$ is a Maxwellian distribution function which has a bump on the Maxwell distribution tail; the physical parameters are the following

$$
n_{p}=0.9, n_{b}=0.2, u=4.5, v_{t}=0.5
$$

whereas the numerical parameters are $N_{x}=128, N_{v}=128, v_{\max }=9$. For the charge conservation algorithms, CFL imposes $\Delta t<0.0545$, so the cases $\mathrm{CFL}<1$ will be made with $\Delta t=0.05$, and CFL $>1$ with $\Delta t=0.2$. We still give the same diagnostics on Fig. 10, 11, 12 and 13. For this electric energy diagnostic, we expect oscillatory behavior of frequency equal to 1.05 ; moreover, since an instability will be declared, the electric energy has to increase up to saturation at $t \approx 20.95$ and to converge for large times (see $[26,32]$ ). The results are really precise for RK and CK, even in long time, under the assumption CFL $<1$ for the charge algorithms developed in section 4 .

On Fig. 14, the $L^{2}$ norm of the distribution function and the entropy $-\int f \ln (f) d x d v$ are plotted for $\Delta t=0.2$. These two quantities are known to be theoretically preserved. As it was already explained, this cannot be reached with our kind of numerical methods. The conservation properties are better for the third order CK3 and RK3 than for the second order CK2 and RK2. Let us precise that more $\Delta t$ increases, more these differences are important, and that the RK algorithms perform a bit better, but are more expensive computationaly speaking. 
Bump on Tail

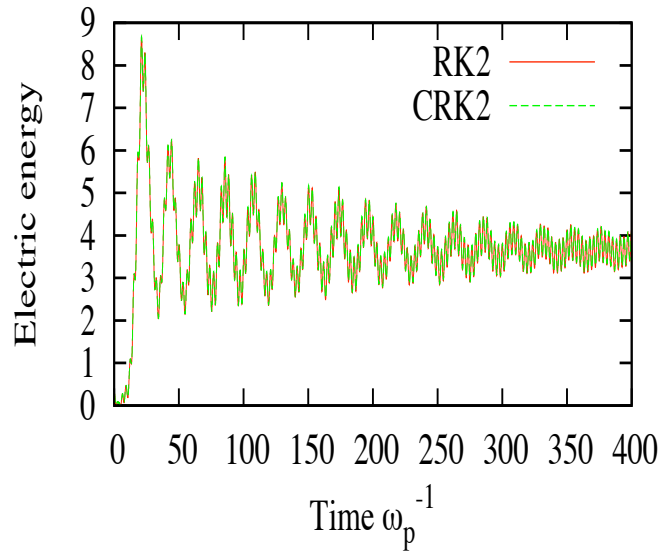

Bump on Tail

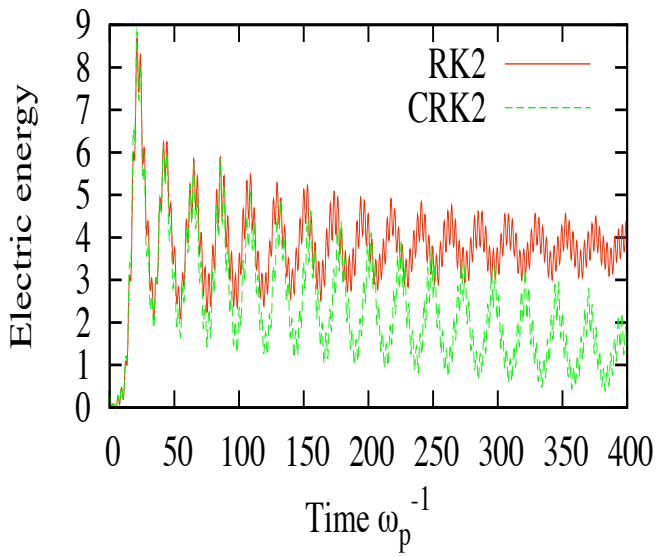

Figure 10: Electric energy $\Delta t=0.05$ (left) $\Delta t=0.2$ (right).
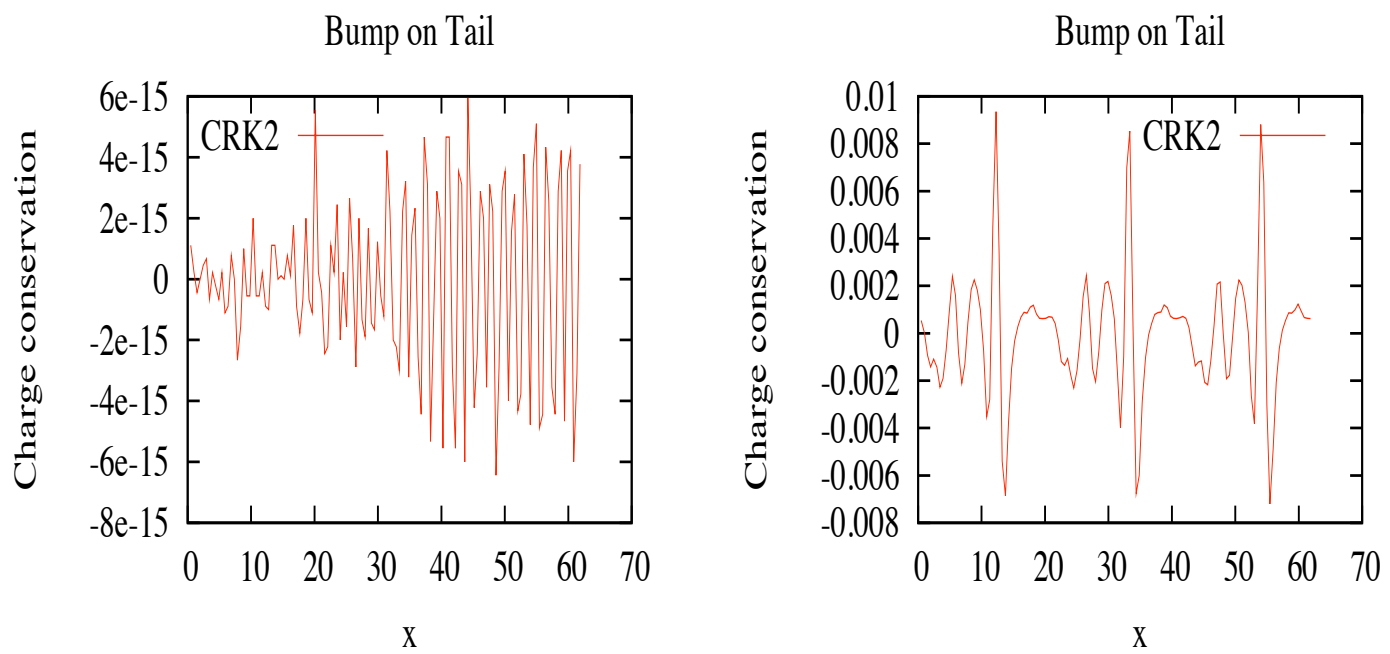

Figure 11: Charge Conservation $\Delta t=0.05$ (left) $\Delta t=0.2$ (right). 


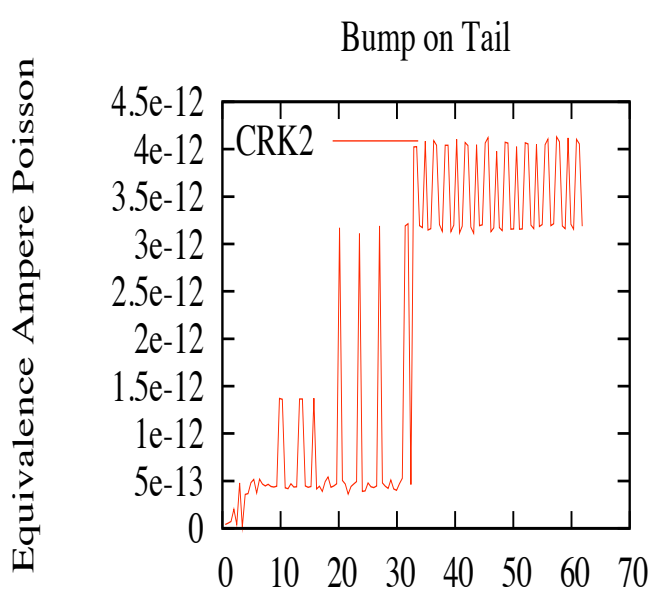

X

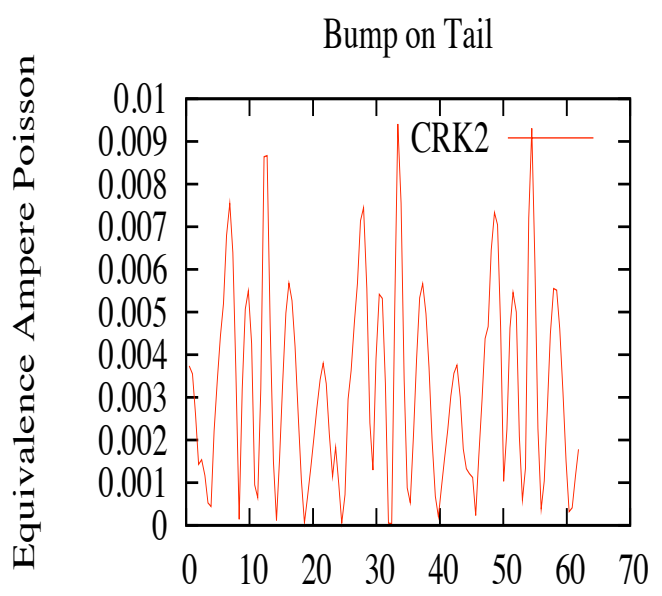

X

Figure 12: Ampère-Poisson equivalence $\Delta t=0.05$ (left) $\Delta t=0.2$ (right).
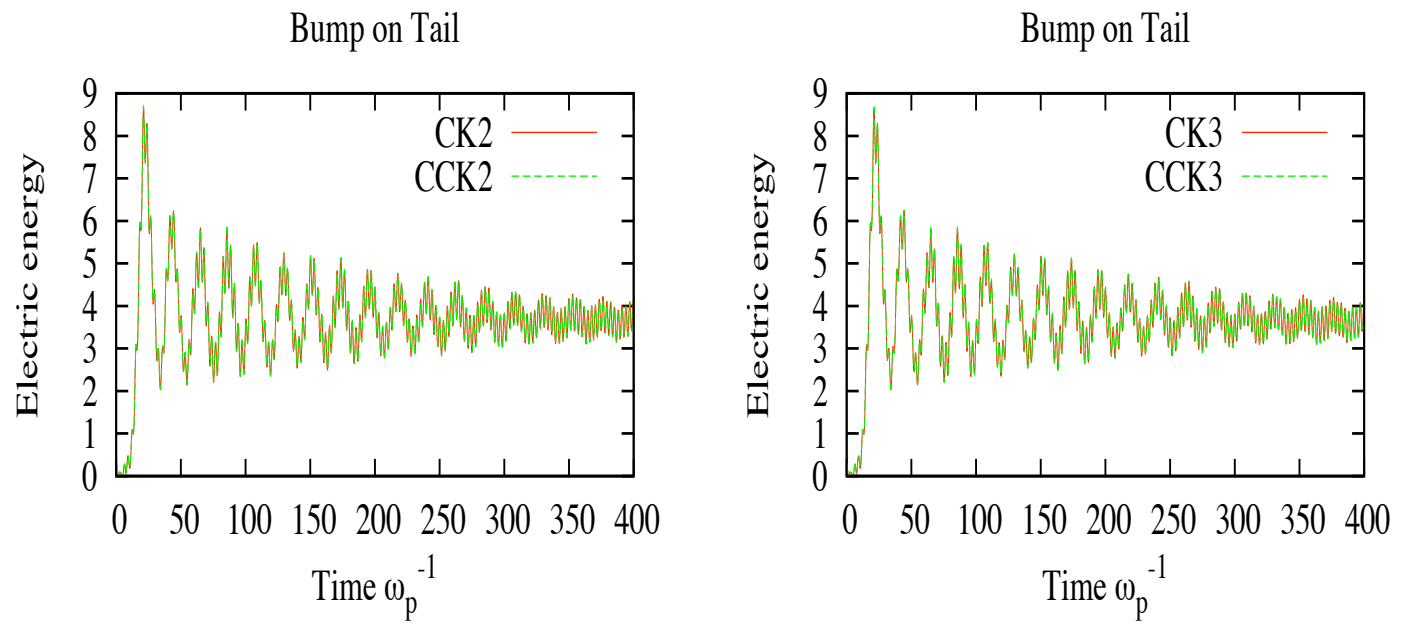

Figure 13: Electric energy $\Delta t=0.05$, CK2 (left), CK3 (right). 

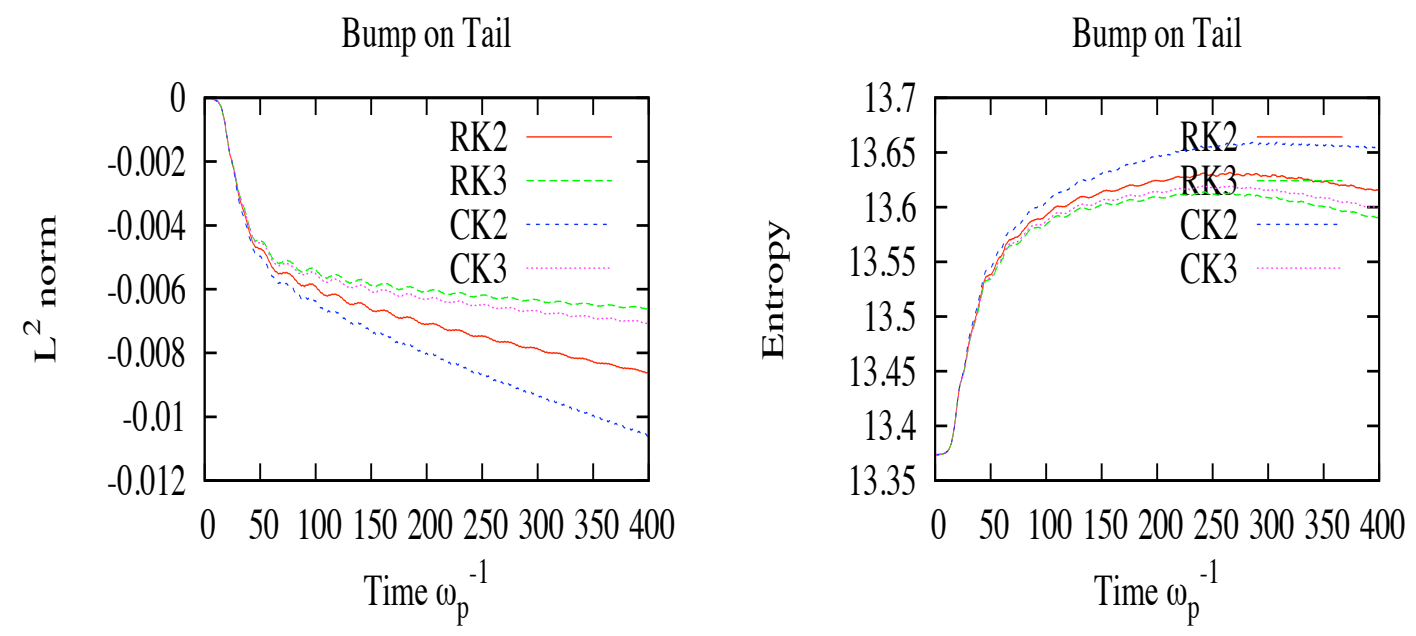

Figure 14: $L^{2}$ norm (left), Entropy (right) $\Delta t=0.2$.

\subsection{QR Vlasov-Maxwell test case}

The numerical method used to solve this test case was presented in section 2.2. Let us precise the numerical parameters used to perform this test case.

$$
f^{0}(x, p)=\frac{1}{\sqrt{2 \pi} T} \exp \left(-\frac{p^{2}}{\left(2 T^{2}\right)}\right)(1+\cos (k x)),
$$

$T=3 k e V$, and $k=\frac{1}{\sqrt{2}}$. A circularly polarized electromagnetic wave is initialized in a periodic domain with a quiver momentum $a_{0}=\sqrt{3}$ :

$$
\begin{array}{cc}
E_{y}^{0}(x)=E_{0} \cos (k x) & E_{z}^{0}(x)=E_{0} \sin (k x) \\
B_{y}^{0}(x)=\frac{-k^{*} E_{0}}{\omega_{0}} \sin (k x) & B_{z}^{0}(x)=\frac{k^{*} E_{0}}{\omega_{0}} \cos (k x) \\
A_{y}^{0}(x)=-\frac{E_{0}}{\omega_{0}} \sin (k x) & A_{z}^{0}(x)=\frac{E_{0}}{\omega_{0}} \cos (k x)
\end{array}
$$

where $k^{*}=k \operatorname{sinc}\left(\frac{k \Delta x}{2}\right)$, and $\operatorname{sinc}(x)=\frac{\sin (x)}{x}$. We consider a pump wave of frequency $\omega_{0}$ and wavenumber $k_{0}$ such that $\omega_{0}^{2}=\omega_{p}^{2} / \gamma_{0}+k_{0}^{2} c^{2}$ is satisfied, with $\gamma_{0}=1+a_{0}^{2}$. Choosing $k_{0} c / m=1 / \sqrt{2}$, we obtain $\omega_{0} / \omega_{p}=1$ (i.e. a ratio $n / n_{c}=1$ ). $\omega_{p}$ is the plasma frequency, $c$ the light velocity and $n_{c}$ the critical density.

These physical parameters correspond to the most unstable mode, for more details about the test case, see $[21,22]$. The numerical parameters are chosen as follows: the impulsion domain is $\left[-p_{\max }, p_{\max }\right]$, where $p_{\max }=8.5$. The choice of $k_{0}$ determines the size of the periodic space domain which is taken equal to $[0,2 \pi \sqrt{2}], N_{x}=256, N_{p}=256, \Delta t=0.01$, which is under CFL condition.

We display on Fig. 15, 16, 17, 18, the distribution function at different times, for a Backward semi-Lagrangian algorithm, which shall be our reference, and our FSL charge conserving algorithm introduced in section 4. The two methods are difficult to compare with only this diagnostic, and seem to behave similarly. On Fig. 19, the mass conservation for BSL and FSL is plotted. FSL behaves far better, especially with long time scaling. Obviously, as the mass decreases, the charge cannot be conserved anymore, which is shown on Fig. 20, 21. Before the decrease of the mass, the 
FSL algorithm preserves charge exactly, and after the decrease, remains truly better. To conclude, on Fig. 22, 23 the integration of $f$ with respect to $x$ is displayed, at different times. At the beginning, the two curves are very close, but as time goes on, the two curves separate, and FSL remains more centered than BSL. For more details about comparisons between FSL and BSL, the reader is refered to [10].

FSL

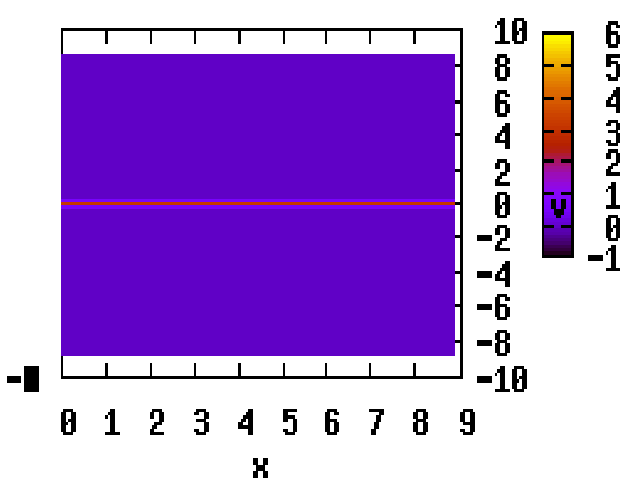

FSL

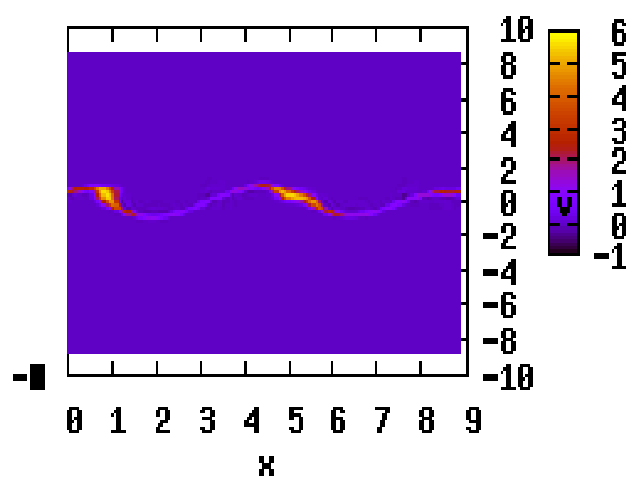

Figure 15: Distribution function at time $=10$ and $22, N_{x}=256, N_{p}=256, \Delta t=0.01$.

BSL

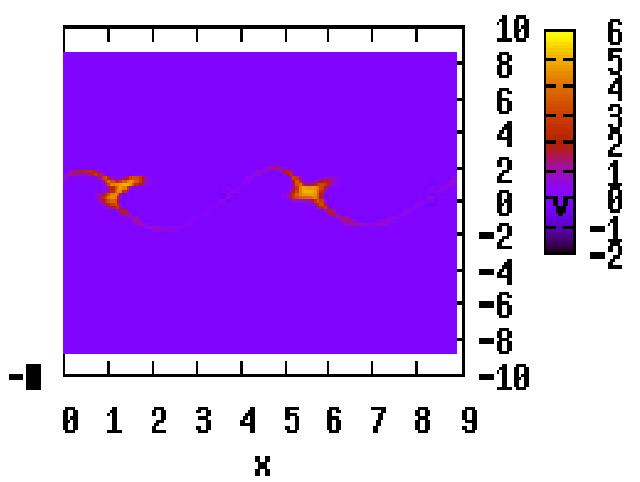

FSL

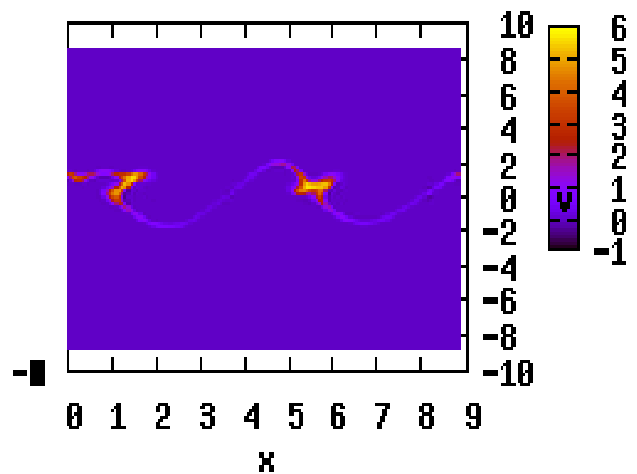

Figure 16: Distribution function at time $=23$, BSL vs FSL, $N_{x}=256, N_{p}=256, \Delta t=0.01$. 
BSL

FSL
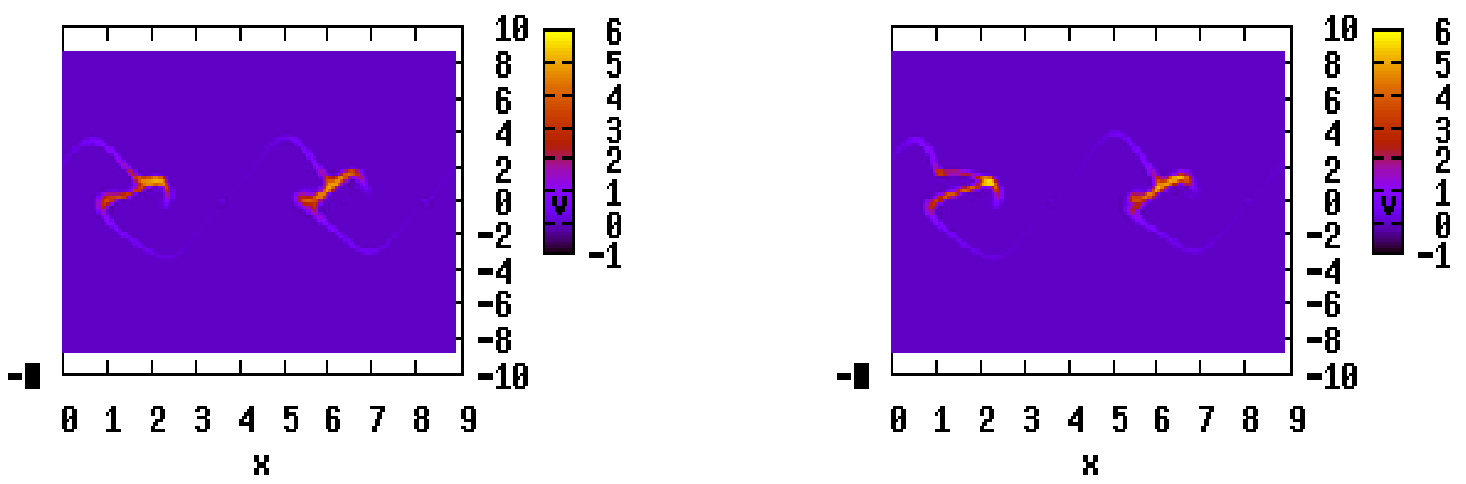

Figure 17: Distribution function at time $=24, \mathrm{BSL}$ vs FSL, $N_{x}=256, N_{p}=256, \Delta t=0.01$.

BSL

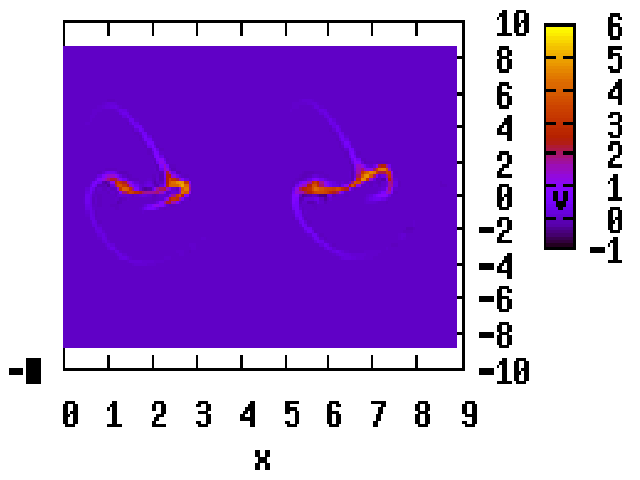

FSL

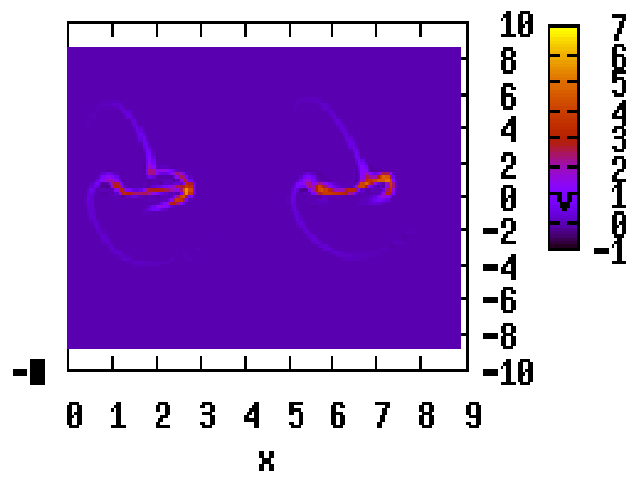

Figure 18: Distribution function at time $=25$, BSL vs FSL, $N_{x}=256, N_{p}=256, \Delta t=0.01$. 


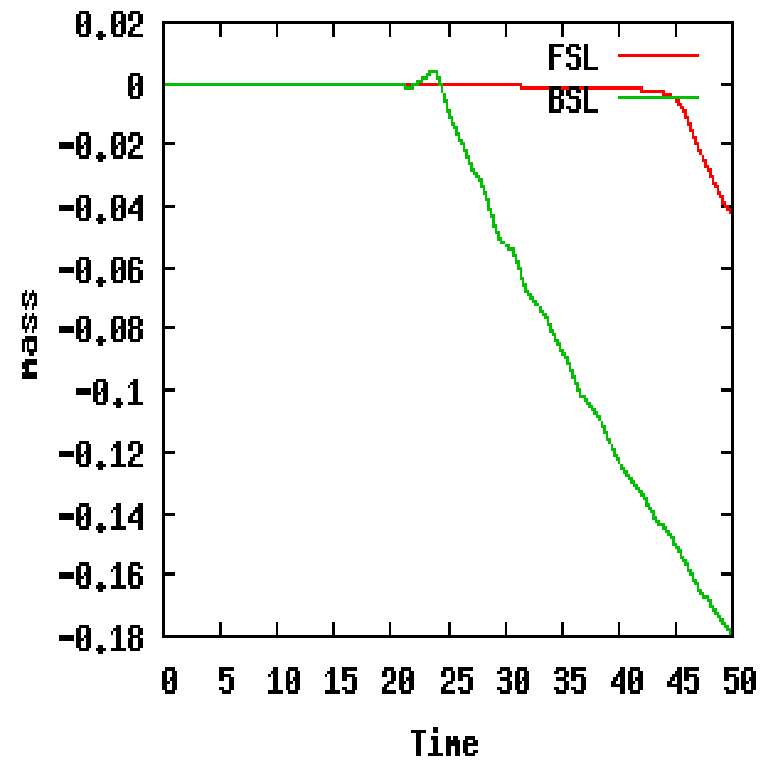

Figure 19: Mass, FSL vs BSL, $N_{x}=256, N_{p}=256, \Delta t=0.01$.
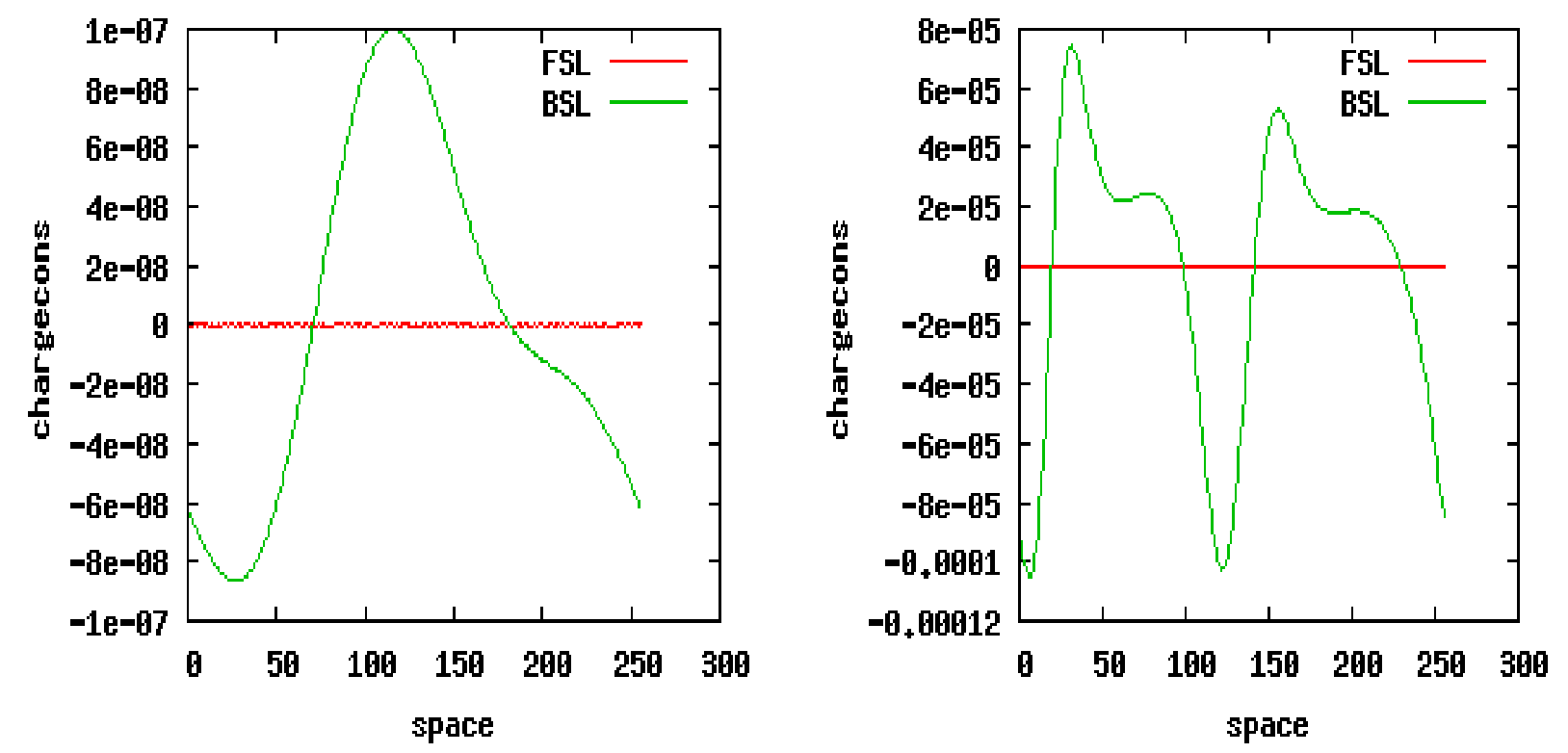

Figure 20: Charge conservation at time 10 and 20, BSL vs FSL, $N_{x}=256, N_{p}=256, \Delta t=0.01$. 

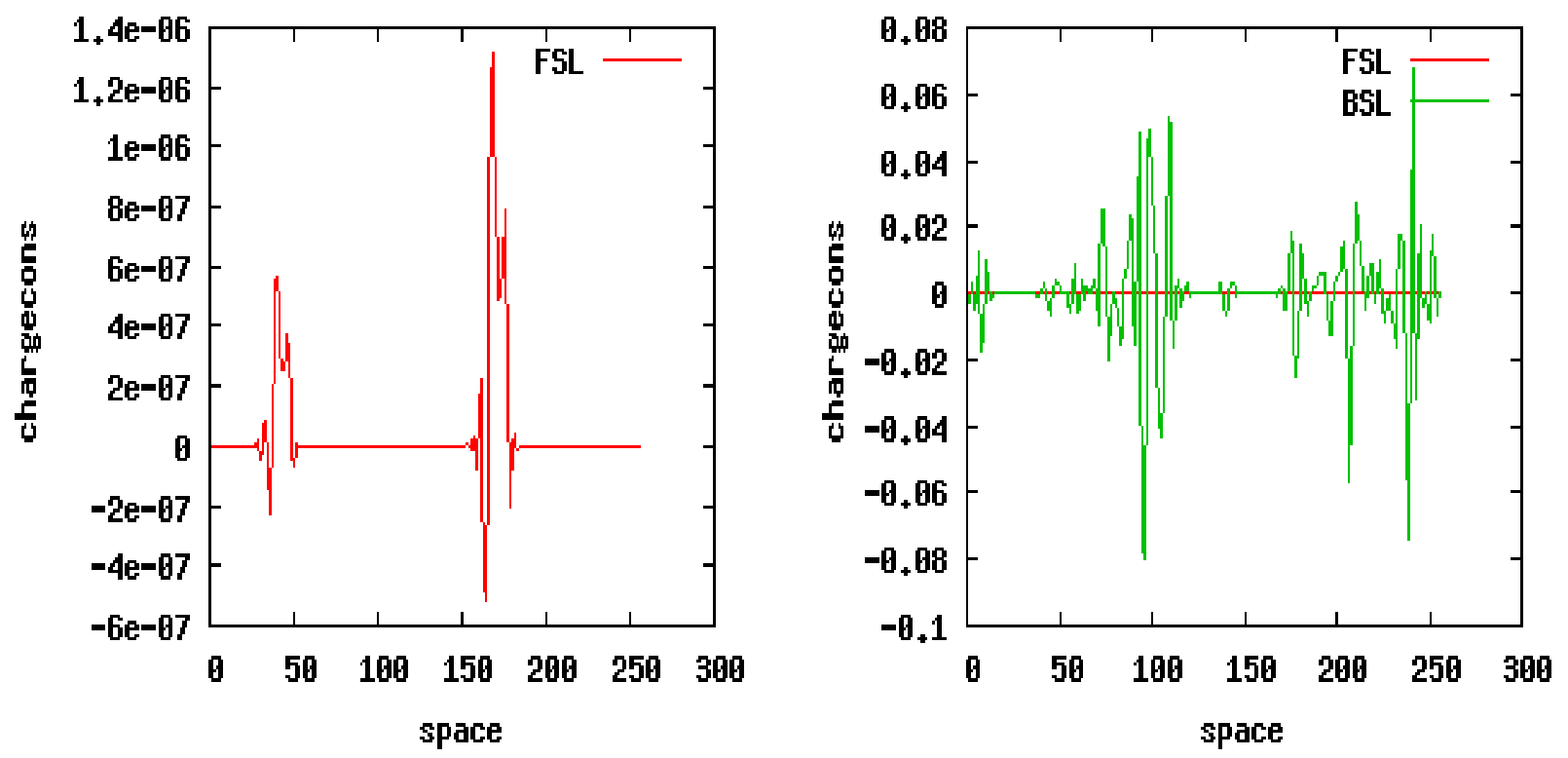

Figure 21: Charge conservation at time 26 and 30, BSL vs FSL, $N_{x}=256, N_{p}=256, \Delta t=0.01$.
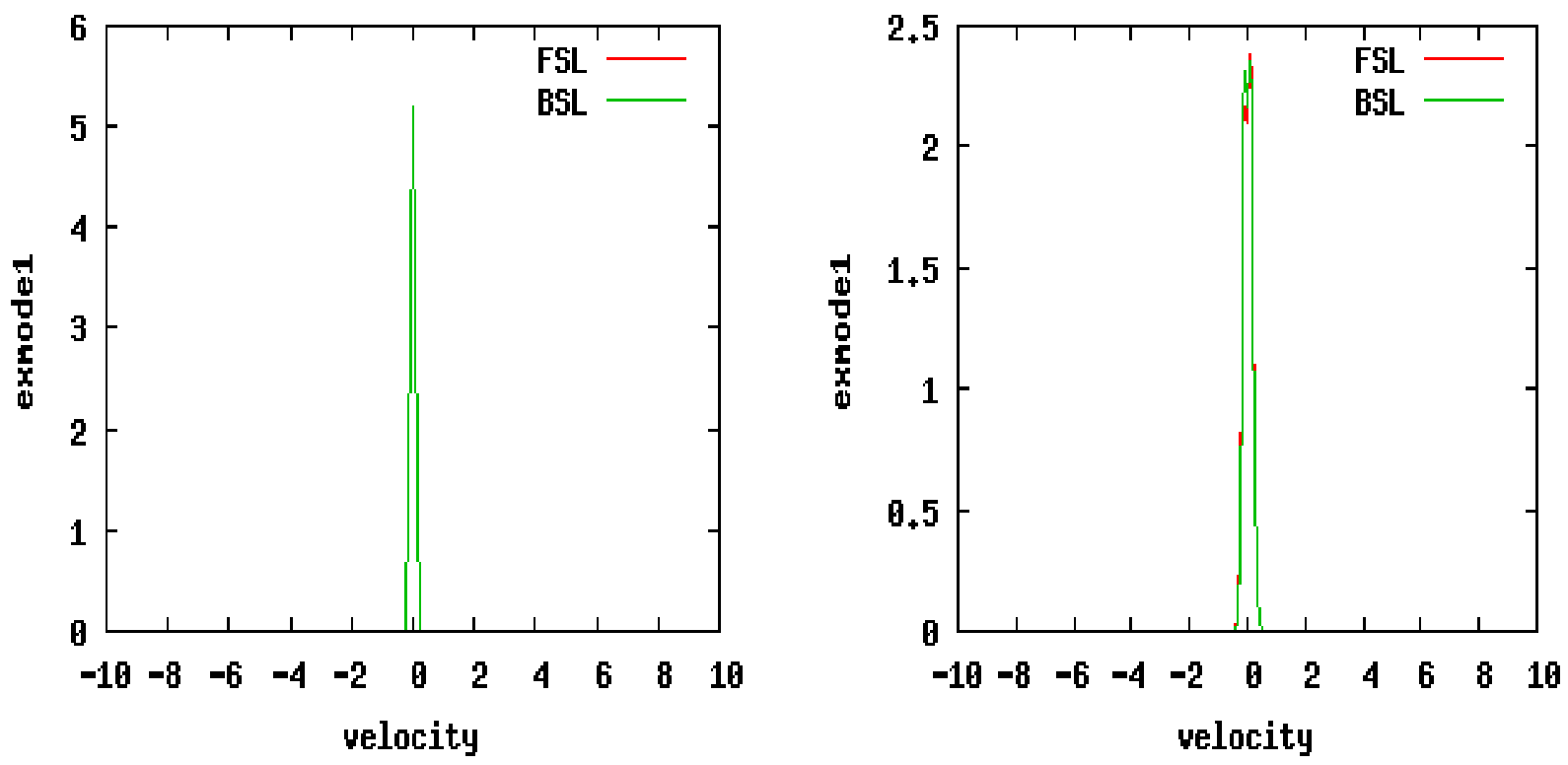

Figure 22: Integration of $f$ along $x, t=1000$ and 2000, BSL vs FSL, $N_{x}=256, N_{p}=256, \Delta t=0.01$. 

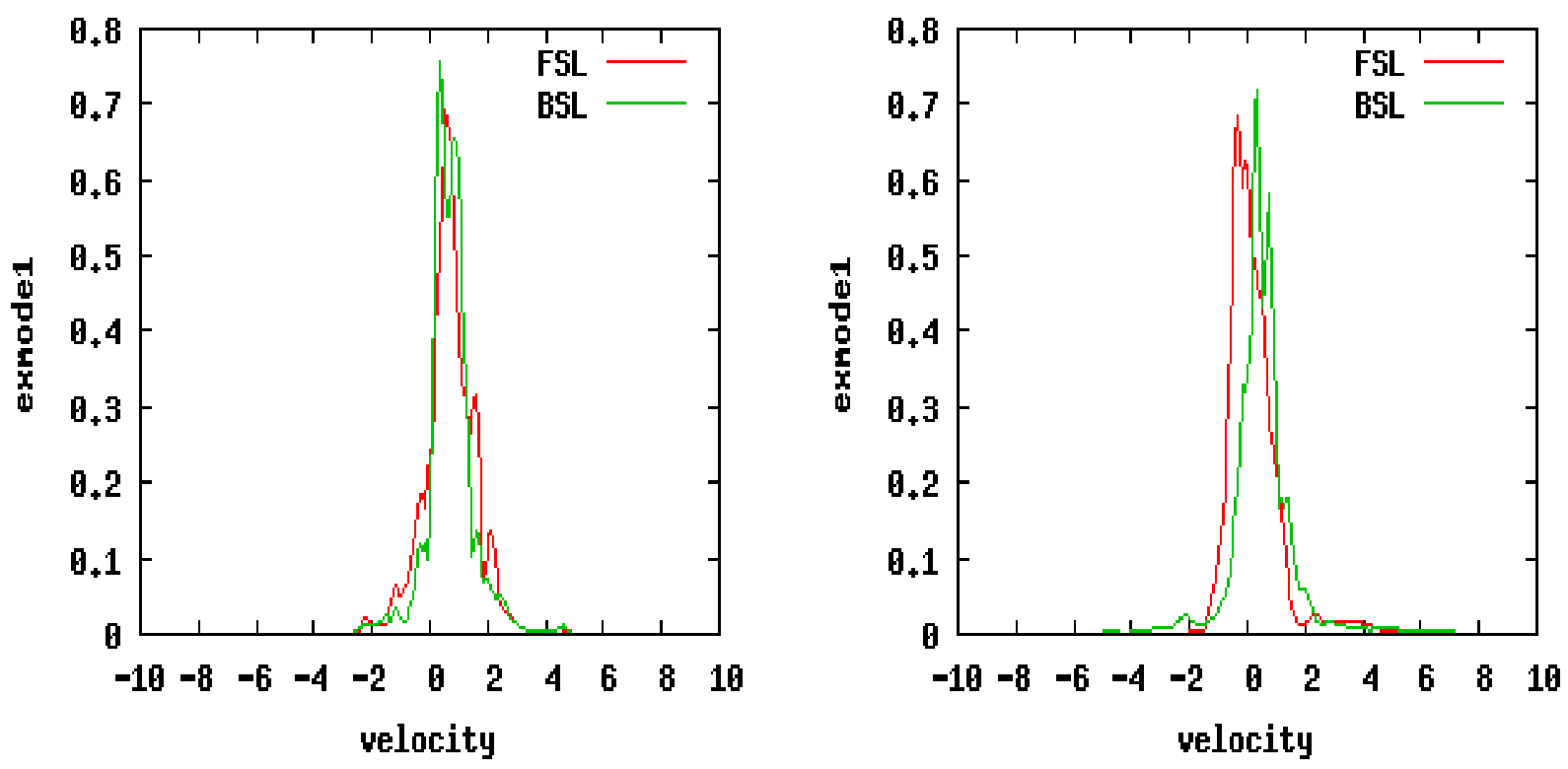

Figure 23: Integration of $f$ along $x, t=30$ and 40, BSL vs FSL $N_{x}=256, N_{p}=256, \Delta t=0.01$.

\subsection{Numerical Synthesis}

One of our goals was to compare these different algorithms in order to choose the best one for a prospective work in view of tackling the $4 \mathrm{D}$ case. First, it has to be said that the CK procedure is faster than the RK one, especially when you look for high order simulation, merely because there are no intermediate deposition steps, which is one of the most expensive step. Obviously, in CCK methods, a $2 \mathrm{D}$ deposition is made to compute $J$, therefore the CPU time is the same as the one of RK (see Table 1). The results concerning electric energy are really difficult to compare, and seem very good whatever algorithm you choose. We observe that the conserved quantities, like the $L^{2}$ norm of the distribution function are a bit better with RK, so CRK seems to be a good compromise between cost and accuracy. These methods are validated by these 1D test cases, and are encouraging for prospective work.

\begin{tabular}{|c||c||c||c||c||c||c||c||c|}
\hline & RK2 & RK3 & Verlet & CK2 & CK3 & CRK2 & CCK2 & CCK3 \\
\hline $64 \times 64$ & $27.1 \mathrm{~s}$. & $42.5 \mathrm{~s}$. & $26 \mathrm{~s}$. & $19 \mathrm{~s}$. & $20.8 \mathrm{~s}$. & $27.6 \mathrm{~s}$. & $27.1 \mathrm{~s}$. & $42.5 \mathrm{~s}$. \\
\hline
\end{tabular}

Table 1: Comparison of the CPU time of the different methods. 10000 iterations.

Moreover, with the last test case, with real Maxwell's equations, we see that a charge conserving FSL method is really better than a classical BSL one, which is entirely satisfying, and convincing for future work.

\section{Conclusion}

In this paper, charge preserving Forward semi-Lagrangian algorithms of second and third order for the characteristics have been developed, and the numerical results meet requirements for various test cases. In these cases, under a CFL condition, the two methods Vlasov-Poisson and VlasovAmpère have proven to be exactly the same, and the charge has proved to be preserved. A future 
work will be to design a 4D charge preserving FSL Vlasov-Maxwell scheme, especially comparing charge preserving algorithms developed in [30] with a procedure derived from this work, using a splitting scheme.

\section{References}

[1] R. Barthelmé, Le problème de conservation de la charge dans le couplage des équations de Vlasov et de Maxwell, Thèse de l'Université Louis Pasteur, 2005.

[2] M. Bostan, N. Crouseilles, Convergence of a semi-Lagrangian scheme for the reduced Vlasov-Maxwell sytem for laser-plasma interaction, Numer. Math. 112, no2, pp. 169-195 (2009).

[3] C.K. Birdsall, A.B. Langdon, Plasma Physics via Computer Simulation, Inst. of Phys. Publishing, Bristol/Philadelphia, (1991).

[4] F. Bouchut, F. Golse, M. Pulvirenti, Kinetic equations and asymptotic theory, Series in applied Math. P.G Ciarlet and P.L Lions (Eds) Gauthier Villars (2008).

[5] J. A. Carrillo, S. Labrunie, Global solutions for the one-dimensional Vlasov-Maxwell system for laser-plasma interaction, Math. Models Methods Appl. Sci. 16, pp. 19-57, (2006).

[6] J.-A. Carrillo, F. Vecil, Non oscillatory interpolation methods applied to Vlasov-based models, SIAM Journal of Sc. Comput. 29, pp. 1179-1206, (2007).

[7] C. Z. Cheng, G. Knorr, The integration of the Vlasov equation in configuration space, J. Comput. Phys, 22, pp. 330-3351, (1976).

[8] P. Colella, P.R. Woodward, The Piecewise Parabolic Method (PPM) for gas-dynamical simulations, J. Comput. Phys. 54 (1984) 174-201.

[9] N. Crouseilles, M. Mehrenberger, E. SonnendrüCKer, Conservative semi-Lagrangian schemes for the Vlasov equation, J. Comput. Phys., 229, no6, pp. 1927-1953 (2010).

[10] N. Crouseilles, T. Respaud, E. Sonnendrucker, A forward semi-Lagrangian method for the numerical solution of the Vlasov equation, Comput. Phys. Comm. 180, pp.1730-1745, (2009).

[11] N. Crouseilles, A. Ghizzo, S. Salmon, Vlasov laser-plasma interaction simulations with a moving grid, INRIA Research Report number 6109 (2007).

[12] C.J. Cotter, J. Frank, S. Reich The remapped particle-mesh semi-Lagrangian advection scheme, Q. J. Meteorol. Soc., 133, pp. 251-260, (2007).

[13] J.W. Eastwood, Virtual particle methods, Comp. Phys. Comm., 64, Issue 2, pp. 252-266, (1991).

[14] N.V. Elkina, J. BüChner, A new conservative unsplit method for the solution of the Vlasov equation, J. Comput. Phys. 213 pp. 862, (2006).

[15] T. ZH. Esirkepov, Exact charge conservation scheme for Particle-in-Cell simulation with an arbitrary form-factor, Comp. Phys. Comm. 135, pp. 144-153, (2001).

[16] F. Filbet, E. Sonnendrücker, P. Bertrand, Conservative numerical schemes for the Vlasov equation, J. Comput. Phys., 172, pp. 166-187, (2001). 
[17] F. Filbet, E. SonnendrüCker, Comparison of Eulerian Vlasov solvers, Comput. Phys. Comm., 151, pp. 247-266, (2003).

[18] A. Ghizzo, P. Bertrand, M.L. Begue, T.W. Johnston, M. Shoucri, A Hilbert-Vlasov code for the study of high-frequency plasma beatwave accelerator, IEEE Transaction on Plasma Science, 24, p. 370, (1996).

[19] A. Ghizzo, P. Bertrand, M. Shoucri, T.W. Johnston, E. Fijalkow, M.R. Feix, A Vlasov code for the numerical simulation of stimulated Raman scattering, J. Comput. Phys. 90, pp. 431-457, (1990).

[20] V. Grandgirard, M. Brunetti, P. Bertrand, N. Besse, X. Garbet, P. Ghendrih, G. Manfredi, Y. Sarrazin, O. Sauter, E. Sonnendrücker, J. Vaclavik, L. Villard, A drift-kinetic semi-Lagrangian $4 D$ code for ion turbulence simulation, J. Comput. Phys., 217, pp. 395-423, (2006).

[21] F. Huot, A. Ghizzo, P. Bertrand, E. Sonnendrücker, O. Coulaud Instability of the time splitting scheme for the one-dimensional and relativistic Vlasov-Maxwell system, J. Comput. Phys., 185, pp. 512-531, (2003).

[22] F. Huot, A. Ghizzo, P. Bertrand, E. Sonnendrücker, O. Coulaud Study of a propagation of ultraintense electromagnetic wave through plasma using semi-Lagrangian Vlasov codes, IEEE Trans. on Plasma SC., 28, (2000).

[23] T.W. Johnston, P. Bertrand, A. Ghizzo, M. Shoucri, E. Fijalkow, M.R. Feix, Simulated Raman scattering: Action evolution and particle trapping via Euler-Vlasov, fluid simulation, Phys. Fluids B4, pp. 2523-2537, (1992).

[24] B. MARDER, A method for incorporating Gauss's law into electromagnetic PIC codes, J. Comput. Phys. 68, pp. 48-55, (1987).

[25] C.-D. Munz, P. Omnes, R. Schneider, E. SonnendrüCker, U. Voss, Divergence Correction Techniques for Maxwell Solvers Based on a Hyperbolic Model, J. Comput. Phys., 161, Issue 2, pp. 484-511, (2000).

[26] T. Nakamura, T. YaBe, Cubic interpolated propagation scheme for solving the hyperdimensional Vlasov-Poisson equation in phase space, Comput. Phys. Comm., 120, pp. 122-154, (1999).

[27] E. Pohn, M. Shoucri, M. Kamelander, Comp. Phys. Comm. 137 , pp. 380-395 (2001).

[28] S. REICH, An explicit and conservative remapping strategy for semi-Lagrangian advection, Atmospheric Science Letters, 8, pp. 58-63, (2007).

[29] T. Respaud, E. SonnendrüCKer Analysis of a new class of Forward semi-Lagrangian schemes for the $1 D$ Vlasov Poisson Equations, HAL: hal-00442957, Submitted.

[30] N.J. Sircombe, T.D. Arber, VALIS: A split-conservative scheme for the relativistic 2D; J. Comput. Phys., 228, pp. 4773-4788 (2009).

[31] M. SHOucRI, A two-level implicit scheme for the numerical solution of the linearized vorticity equation, Int. J. Numer. Meth. Eng., 17, p. 1525 (1981).

[32] M. Shoucri, Nonlinear evolution of the bump-on-tail instability, Phys. Fluids, 22, p. 20382039 (1979). 
[33] H. Schmitz, R. Grauer, Comparison of time splitting and backsubstitution methods for integrating Vlasov's equation with magnetic fields, Comput. Phys. Commun. 175, pp.86 (2006).

[34] E. Sonnendrücker, J. Roche, P. Bertrand, A. Ghizzo, The semi-Lagrangian method for the numerical resolution of the Vlasov equation, J. Comput. Phys., 149, pp. 201-220, (1999).

[35] T. Umeda, Y. Omura, T. Tominaga, H. Matsumoto, A new charge conservation method in electromagnetic particle-in-cell simulations, Comp. Phys. Comm 156, Issue1, pp. 73-85, (2003).

[36] J. Villasenor, O. Buneman, Rigorous charge conservation for local electromagnetic field solvers, Comp. Phys. Comm. 69, pp. 306-316, (1992).

[37] M. Zerroukat, N. Wood, A. Staniforth, A monotonic and positive-definite filter for a Semi-Lagrangian Inherently Conserving and Efficient (SLICE) scheme, Q.J.R. Meteorol. Soc., 131, pp 2923-2936, (2005).

[38] M. Zerroukat, N. Wood, A. Staniforth, The Parabolic Spline Method (PSM) for conservative transport problems, Int. J. Numer. Meth. Fluids, 51, pp. 1297-1318, (2006). 